\title{
Next generation haplotyping to decipher nuclear genomic interspecific admixture in Citrus species: analysis of chromosome 2
}

Franck Curk ${ }^{1,2 \dagger}$, Gema Ancillo ${ }^{2 \dagger}$, Andres Garcia-Lor ${ }^{2}$, François Luro ${ }^{1}$, Xavier Perrier ${ }^{3}$, Jean-Pierre Jacquemoud-Collet ${ }^{3}$, Luis Navarro ${ }^{2^{*}}$ and Patrick Ollitrault ${ }^{2,3^{*}}$

\begin{abstract}
Background: The most economically important Citrus species originated by natural interspecific hybridization between four ancestral taxa (Citrus reticulata, Citrus maxima, Citrus medica, and Citrus micrantha) and from limited subsequent interspecific recombination as a result of apomixis and vegetative propagation. Such reticulate evolution coupled with vegetative propagation results in mosaic genomes with large chromosome fragments from the basic taxa in frequent interspecific heterozygosity. Modern breeding of these species is hampered by their complex heterozygous genomic structures that determine species phenotype and are broken by sexual hybridisation. Nevertheless, a large amount of diversity is present in the citrus gene pool, and breeding to allow inclusion of desirable traits is of paramount importance. However, the efficient mobilization of citrus biodiversity in innovative breeding schemes requires previous understanding of Citrus origins and genomic structures. Haplotyping of multiple gene fragments along the whole genome is a powerful approach to reveal the admixture genomic structure of current species and to resolve the evolutionary history of the gene pools. In this study, the efficiency of parallel sequencing with 454 methodology to decipher the hybrid structure of modern citrus species was assessed by analysis of 16 gene fragments on chromosome 2 .

Results: 454 amplicon libraries were established using the Fluidigm array system for 48 genotypes and 16 gene fragments from chromosome 2. Haplotypes were established from the reads of each accession and phylogenetic analyses were performed using the haplotypic data for each gene fragment. The length of 454 reads and the level of differentiation between the ancestral taxa of modern citrus allowed efficient haplotype phylogenetic assignations for 12 of the 16 gene fragments. The analysis of the mixed genomic structure of modern species and cultivars (i) revealed C. maxima introgressions in modern mandarins, (ii) was consistent with previous hypotheses regarding the origin of secondary species, and (iii) provided a new picture of the evolution of chromosome 2.
\end{abstract}

Conclusions: 454 sequencing was an efficient strategy to establish haplotypes with significant phylogenetic assignations in Citrus, providing a new picture of the mixed structure on chromosome 2 in 48 citrus genotypes.

Keywords: Phylogeny, Haplotype, Evolution, SNP, NGS, Genome admixture

\footnotetext{
*Correspondence: Inavarro@ivia.es; ollitrault@cirad.fr

${ }^{\dagger}$ Equal contributors

${ }^{2}$ Centro de Protección Vegetal y Biotecnología, Instituto Valenciano de Investigaciones Agrarias (IVIA), 46113 Moncada, Valencia, Spain

${ }^{3}$ UMR AGAP, Centre de coopération Internationale en Recherche Agronomique pour le Développement (CIRAD), TA A-108/02, 34398

Montpellier, Cedex 5, France

Full list of author information is available at the end of the article
}

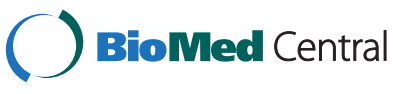

(c) 2014 Curk et al.; licensee BioMed Central. This is an Open Access article distributed under the terms of the Creative Commons Attribution License (http://creativecommons.org/licenses/by/4.0), which permits unrestricted use, distribution, and reproduction in any medium, provided the original work is properly credited. The Creative Commons Public Domain Dedication waiver (http://creativecommons.org/publicdomain/zero/1.0/) applies to the data made available in this article, unless otherwise stated. 


\section{Background}

World-wide production of citrus was 131 million tonnes in 2011 and 2012 [1]. The main citrus varietal groups are sweet oranges (52\%), small citrus (21\%), limes and lemons (12\%), and grapefruits and pummelos (6\%). The inter-varietal genetic diversity of most of these varietal groups is very scarce, particularly for sweet oranges, lemons, and grapefruits, where intra-group diversity results from clonal variation/selection in vegetatively propagated material [2]. This confers a substantial fragility of these groups against emerging diseases, as demonstrated by the ongoing major crisis in the Brazilian and Floridian citrus industries [3-5]. Moreover, conventional breeding of these species is hampered by their complex heterozygous genomic structures that determine species phenotype and are broken by sexual hybridisation. Therefore, most breeding efforts for sweet orange, grapefruit, and lemons to date have used natural or induced mutations and somaclonal variation [6]. However, important natural phenotypically useful variability exists in the citrus gene pool particularly for resistance to biotic and abiotic constraints [7]. The efficient mobilization of this biodiversity in innovative breeding schemes will require prior knowledge of varietal group origins and genomic structures.

The taxonomy of Citrus remains controversial due to the conjunction of broad morphological diversity, total interspecific sexual compatibility within the genus, and partial apomixis of many cultivars. Fixing complex genetic structures through seedling propagation via apomixis led some taxonomists to consider clonal families of interspecific origin as new species [8]. Two major systems are widely used to classify Citrus species: the Swingle and Reece [9] classification, which identifies 16 species, and the Tanaka [10] classification, which recognizes 156 species. More recently, Mabberley [11] proposed a new classification of edible citrus that recognized three species and four hybrid groups. In this paper, we will use the Swingle and Reece [9] classification system. This taxonomic system is widely used in the citrus scientific community and, as mentioned below, mostly agrees with molecular data. Despite the difficulties involved in establishing a consensus classification system for edible citrus, most authors now agree on the origins of the main cultivated forms. Molecular analyses clarified the genetic underpinnings of various cultivated species of Citrus [12-18]. Four ancestral taxa [C. medica L. (citron), C. reticulata Blanco (mandarin), C. maxima (Burm.) Merr. (pummelo), and C. micrantha Wester (papeda)] were identified as the ancestors of all cultivated Citrus $[13,15]$. Differentiation between these sexually compatible taxa may be explained by foundation effects in three distinct geographic zones and by an initial allopatric evolution. C. maxima originated in the Malay
Archipelago and Indonesia, C. medica evolved in northeastern India and the nearby region of Myanmar and China, and C. reticulata diversification occurred over a region including Vietnam, southern China, and Japan $[8,19]$. Secondary species [C. sinensis (L.) Osb. (sweet orange), C. aurantium L. (sour orange), C. paradisi Macf. (grapefruit), C. limon (L.) Burm. (lemon), and C. aurantifolia (Christm.) Swing. (lime)] arose from hybridizations between the four basic taxa $[13,15]$. Partial apomixis of most of the secondary species has been an essential element in the limitation of the number of further interspecific meiosis. Moreover, studies considering diversity of morphological characteristics [20,21], primary metabolites [22], and secondary metabolites [23] confirmed that a major part of the phenotypic diversity of edible citrus resulted from differentiation between the basic taxa. In this context, deciphering the phylogenomic structures of the secondary citrus species is essential before innovative conventional breeding strategies can be developed.

Reticulations pose serious challenges in phylogenetic analyses and result in evolutionary histories that cannot be adequately represented in the form of phylogenetic trees [24-28]. For many species, these relationships resemble a network with phylogenetic incongruities observed not only between cytoplasmic and nuclear genomes, but also between different regions of nuclear genomes [29-32]. In plants such as citrus, where vegetative propagation such as apomixis took place immediately or a few generations after a reticulation event, large parts of the genome remain in interspecific heterozygosity. Genome-wide molecular analyses are, therefore, needed to decipher the complex interspecific mosaic genomes resulting from such evolution. Studies based on linkage disequilibrium can provide good evidence for recent and ancient hybridization events. This was demonstrated in sunflower by Rieseberg et al. [33,34], who showed that the genomes of hybrid sunflower species contained chromosomal segments from both parental species. When examining heterozygous structures like citrus genotypes, phased multilocus studies offer improvements over monolocus analysis for the identification of interspecific heterozygous genome fragments deriving from reticulate events. The expectation is that tightly linked markers in a hybrid species are significantly more likely to come from the same parent and, therefore, to display linkage disequilibrium [29]. Sanger sequencing after bacterial cloning to separate gene copies was used effectively for such analysis [35-37]. However, because this is time-consuming and expensive, and only a few individuals and genes can be investigated, this type of analysis can miss intraspecific diversity components and may lead to erroneous conclusions about the evolutionary history of related taxa [38]. In recent years, massively parallel sequencing of barcoded DNA mixtures enabled rapid and 
relatively inexpensive DNA sequence data production and facilitated genome-wide sequence variant discovery. This analysis was applied to a wide variety of bacteria, fungi [39,40], multi-copy genes [41], and polyploids. In citrus, recent whole genome sequencing projects $[42,43]$ confirmed hybridization at the origin of $C$. sinensis and $C$. clementina (clementine) and allowed the phylogenetic origin of DNA fragments in the whole genome to be determined. However, the genomic structure of other secondary species and most modern varieties remain to be studied, and no analysis of the phylogeny of DNA fragments from the whole genome has yet been undertaken.

Whole genome sequencing (WGS) in large populations remains costly and requires considerable bioinformatic analysis. Major challenges include the need to reduce genome complexity and manage orthologous sequence data for a large number of individuals. Alternatives such as targeted capture [44] or targeted amplicon [45] sequencing can be valuable. In human research, deep amplicon sequencing using 454 technology yielded thousands of haplotype calls per amplicon at the beta-defensin locus, and this was considered to be an efficient method for haplotyping and copy-number estimation in small to medium-sized cohorts [41]. A particular advantage of using such an approach for haplotyping heterozygous structures is that sequencing data come from single DNA molecules, and there is no requirement for cloning. Therefore, we hypothesize that, by using a sequencing method allowing enough long reads (over $500 \mathrm{bp}$ ) such as 454 pyrosequencing [41], it should be possible to establish multilocus haplotypes that are phylogenetically significant when working at a sufficient level of genetic differentiation between taxa.
The objective of this work was to analyze the potential of the 454 sequencing method for efficient targeted parallel haplotyping to decipher complex interspecific genomic structures resulting from reticulate evolution in citrus. Amplicons from 48 genotypes, representative of Citrus ancestral taxa and secondary species, were subjected to parallel sequencing. Sixteen targeted genes distributed across chromosome 2 were sequenced. Chromosome 2 was selected due to its complex admixture structure in sweet orange, as identified in our previous research $[16,43]$.

\section{Methods}

Plant material

Leaves from 48 accessions of the Citrus genus and one accession of Severinia buxifolia [Poir.] Tenore were collected from the IVIA Citrus Germplasm Bank of pathogen-free plants (Valencia, Spain; accessions with IVIA identification number) and the INRA/CIRAD Citrus collection of San Giuliano (Corsica, France; accessions with SRA identification number) [Additional file 1]. In addition, in silico data were mined (phytozome.net [46]) from the haploid clementine used to establish the first high-quality reference sequence of Citrus [43].

The Swingle and Reece [9] botanical classification for scientific names was adopted (Table 1 and [Additional file 1]). The four ancestral taxa of the Citrus genus were represented by 31 accessions: 14 mandarins ( 12 C. reticulata and two C. tachibana (Mak.) Tan.), ten pummelos (C. maxima), six citrons (C. medica), and one papeda (C. micrantha). Representatives of secondary citrus species or genotypes included two diploid clementines (C. reticulata), the haploid clementine used to establish the whole citrus genome reference sequence ( $C$. reticulata), three sweet

Table 1 Scientific names and number of accessions per common horticultural group

\begin{tabular}{|c|c|c|c|}
\hline & $\begin{array}{l}\text { Common horticultural } \\
\text { group name }\end{array}$ & Swingle scientific name & $\begin{array}{l}\text { Number of } \\
\text { accessions }\end{array}$ \\
\hline \multirow[t]{5}{*}{ Ancestral groups } & Pummelo & Citrus maxima (Burm.) Merr. & 10 \\
\hline & Mandarin & Citrus reticulata Blanco & 12 \\
\hline & & Citrus tachibana (Mak.) Tan. & 2 \\
\hline & Citron & Citrus medica L. & 6 \\
\hline & Papeda & Citrus micrantha Wester & 1 \\
\hline \multirow{8}{*}{$\begin{array}{l}\text { Secondary species or genotypes arising from } \\
\text { hybridizations between ancestral groups }\end{array}$} & Bergamot & Citrus aurantifolia (Christm.) Swing. & 1 \\
\hline & Lime & Citrus aurantifolia (Christm.) Swing. & 1 \\
\hline & Alemow & Citrus aurantifolia (Christm.) Swing. & 1 \\
\hline & Sour orange & Citrus aurantium L. & 2 \\
\hline & Lemon & Citrus limon (L.) Burm. & 5 \\
\hline & Grapefruit & Citrus paradisi Macf. & 2 \\
\hline & Clementine & Citrus reticulata Blanco & 3 \\
\hline & Sweet orange & Citrus sinensis (L.) Osb. & 3 \\
\hline Out-group & & Severinia buxifolia (Poir.) Ten. & 1 \\
\hline
\end{tabular}


oranges (C. sinensis), two sour oranges (C. aurantium), two grapefruits (C. paradisi), five lemons (C. limon), one bergamot ( $C$. aurantifolia), one lime $(C$. aurantifolia), and one 'Alemow' ( $C$. aurantifolia). These 18 genotypes were putative hybrids derived from the four ancestral taxa. One Citrus genus relative (Severinia buxifolia) was added as an out-group.

\section{DNA extraction}

High molecular weight genomic DNA was extracted from leaf samples using the DNeasy Plant Mini Kit (Qiagen S.A.; Madrid, Spain) according to the manufacturer's instructions.

\section{Target genomic fragment selection \\ Chromosome 2 targeted genomic fragments}

The reference citrus whole genome sequence, released in Phytozome [46] by the International Citrus Genome Consortium (ICGC), was used to select gene fragments in this study. The annotated genes file ("Cclementina 182_gene.gff3" file) was used and is available at the Phytozome web page [46].

Duplicated and overlapping genes were discarded. SSRs were annotated (up to tetranucleotidic motifs and at least 11 bp sequences) and all genes presenting microsatellite motifs were eliminated. Finally, the genes were sorted by length, and 415 genes were selected, each with a length of 1000-2000 bp. This length was selected to facilitate the design of primers for efficient sequencing of 500-600 bp amplicons. Sixteen genes within chromosome 2 were chosen.

\section{Amplicon library preparation}

For the 16 selected gene fragments of chromosome 2 [Additional file 2], 16 primer pairs were designed (according to the Access Array $^{\text {tm }}$ System for 454 Sequencing Platform User Guide [47]) and loaded on the Fluidigm Access Array. This method employed the same approach as the two-step PCR methods proposed by Bybee et al. [45] and validated by Curk et al. [48] for citrus. Two successive PCR reactions produced amplicons with specific multiplex identifiers (MIDs) and directional titanium primer sequences for each variety. PCR products were generated using a 48.48 Access Array IFC (Fluidigm 48.770 Digital PCR Workflow Quick Reference Card), and amplicon quality was checked using an Agilent 2100 Bioanalyzer (Agilent DNA 1000 Kit Guide). Next, equal volumes of the PCR products were pooled together to create one PCR product library. The PCR product library was purified using AMPure beads. After purification, the PCR product library was quantified using Quant-iT PicoGreen fluorimetry (Quant-iT ${ }^{\mathrm{mi}}$ PicoGreen $^{\circ}$ User Guide) before proceeding to emulsion PCR.
454 parallel sequencing was performed using a mixture of all the amplicons for all the genotypes. DNA from each genotype carried a different MID, as defined by Roche [49]. The 454 sequencing technique requires amplicon primers to contain a directional GS FLX Titanium primer sequence (which includes a four base library "key" sequence) at the $5^{\prime}$ end of the oligonucleotide in addition to the gene-specific sequence at the $3^{\prime}$ end. To allow for automated software identification of samples after pooling and sequencing, MID sequences [Additional file 3] were added between primer A (or B) and the gene-specific sequences [50].

Forty-eight DNA samples were amplified and parallelsequenced on a GS FLX Titanium system (Roche 454). Haploid clementine gene fragment sequences were obtained from the reference citrus whole genome sequence (Phytozome [46]). S. buxifolia (out-group) gene fragments were obtained by PCR amplification performed using a Mastercycler Ep gradient S thermocycler (Eppendorf). PCR was conducted in a final volume of $25 \mu \mathrm{l}$ containing 0.027 $\mathrm{U}$ Taq DNA polymerase (Fermentas), $1 \mathrm{ng} / \mu \mathrm{l}$ of genomic DNA, $10 \times$ PCR buffer (Fermentas), $0.2 \mathrm{mM}$ of each dNTP, $1.5 \mathrm{mM} \mathrm{MgSO}_{4}$, and $0.2 \mu \mathrm{M}$ of each primer. The following PCR program was applied: denaturation at $94^{\circ} \mathrm{C}$ for $5 \mathrm{~min}$; 40 cycles of $30 \mathrm{~s}$ at $94^{\circ} \mathrm{C}, 1 \mathrm{~min}$ at $55^{\circ} \mathrm{C}$, and $2 \mathrm{~min}$ at $72^{\circ} \mathrm{C}$; and a final elongation step of $4 \mathrm{~min}$ at $72^{\circ} \mathrm{C}$. PCR product purification was performed using a QIAquick PCR purification kit (Qiagen S.A.). Amplicons were sequenced using the Sanger method from the $5^{\prime}$ end using fluorescently labeled dideoxynucleotides (Big Dye Terminator Cycle Sequencing Kit v3.1).

\section{Sequencing and sequence data analysis for SNP calling}

Raw reads obtained from 454 pyrosequencing were preprocessed by removal of low-quality reads and adapter/primer sequences using PRINSEQ [51]. Short reads ( $<150$ bases) with primer dimers were considered to be low-quality reads. Remaining reads were automatically identified and sorted by MID and specific gene primers using the SFF Tool commands of Newbler software [52].

For each variety, 454 pyrosequencing reads were aligned independently for each gene using SeqMan NGen software version 7.0 [53] with the following assembly parameters: match size, 12; minimum match percentage, 80; and minimum sequence length, 150. For each gene fragment, consensus alignments from a homozygous sequence comprised one haplotype, while those from a heterozygous sequence comprised two haplotypes.

\section{Genetic analysis of SNP data}

Unbiased expected heterozygosity (He), observed heterozygosity $(\mathrm{Ho})$, fixation index values $\left(\mathrm{F}_{\mathrm{W}}[54]\right)$, and $\mathrm{F}_{\text {Stat }}$ parameters $\left(\mathrm{F}_{\mathrm{ST}}\right.$ and $\mathrm{G}_{\mathrm{ST}}$ Index) were calculated using GENETIX v. 4.03 software [55]. SNP number and location 
were identified with SNiPlay online software $[56,57]$. Principal component analysis (PCA) was performed using XLSTAT software.

Haplotype and genotypic phylogenetic relationships were studied by (i) neighbor-joining analysis (NJA), based on the SNP data using DARwin software [58] with a simple matching dissimilarity index, (ii) maximum likelihood phylogenetic analysis using Mega software [59]. The simple matching dissimilarity index was also used to infer intra- and inter-taxa average differentiation.

Graphical visualization of chromosome 2 genotypes was constructed using GGT2 software [60].

Population structure was inferred using Structure (version 2.3.4) software [61], which implements a modelbased clustering method using genotype data $[62,63]$. No a priori population structure was defined. The linkage model option was used, with allele frequencies correlated and compute probability of the data for estimating K. Analyses were made with K-values (number of subpopulations) of 1-10. The statistics used to select the correct K-value were those used by Evanno et al. [64]. Ten runs using Structure software were performed, each with 50,000 steps of burning followed by 50,000 Monte Carlo Markov Chain (MCMC) repetitions using the linkage model, knowing Map distances between loci [Additional file 2] [17]. The independent Structure-run cluster outputs were permuted and aligned to match one another as closely as possible.

\section{Results}

\section{Read distribution}

The first round of Fluidigm amplification/454 sequencing produced 64,170 reads. Of these, $11 \%$ were short reads with primer dimers, and 57,394 reads were therefore considered useful. Useful reads were classified according to their MID and titanium sequences, and MID sequences were removed using 454 software tools. All reads were attributed to one of the $768(48 \times 16)$ amplicons according to the fragment gene sequence. The average number of reads per amplicon was 75; however, the distribution of reads per amplicon (Figure 1) was asymmetric, resulting in a high proportion of amplicons with insufficient coverage. Based on 454 single-read sequencing data error rates and our preliminary unpublished data, we defined a threshold level of 50 reads per amplicon for confident genotype calling. However, 305 amplicons (40\%) had fewer than this initial threshold number. Detailed analysis of read distribution for each amplicon [Additional file 4] showed that much of the heterogeneity was due to global under-representation of three gene fragments and overrepresentation of five fragments. The total number of reads per variety was less heterogeneous than one per gene fragment. We therefore conducted a second round of Fluidigm/454 sequencing. A total of 159,490 useful reads was obtained (average 208 reads per amplicon) from the combination of the two runs [Additional file 5]. The distribution of the number of reads per amplicon remained highly heterogeneous, and 135 amplicons (18\% of the total gene fragments/varieties) still had fewer than 50 associated reads. In cases where number or quality of reads was insufficient for genotype calling, amplicons were Sanger sequenced to complete the genotypic data set. Sanger sequence analysis also allowed inference of haplotype if only one or no heterozygous loci were observed in the Sanger sequence [Additional file 5].

\section{Genotype calling and polymorphism of gene fragments}

A total of 318 SNPs were identified from 7895 bp readable sequences for the 16 gene fragments within the 48 Citrus accessions (Table 2). The web based SNiPlay tool $[56,57]$ was used to analyze the intragenic location and potential

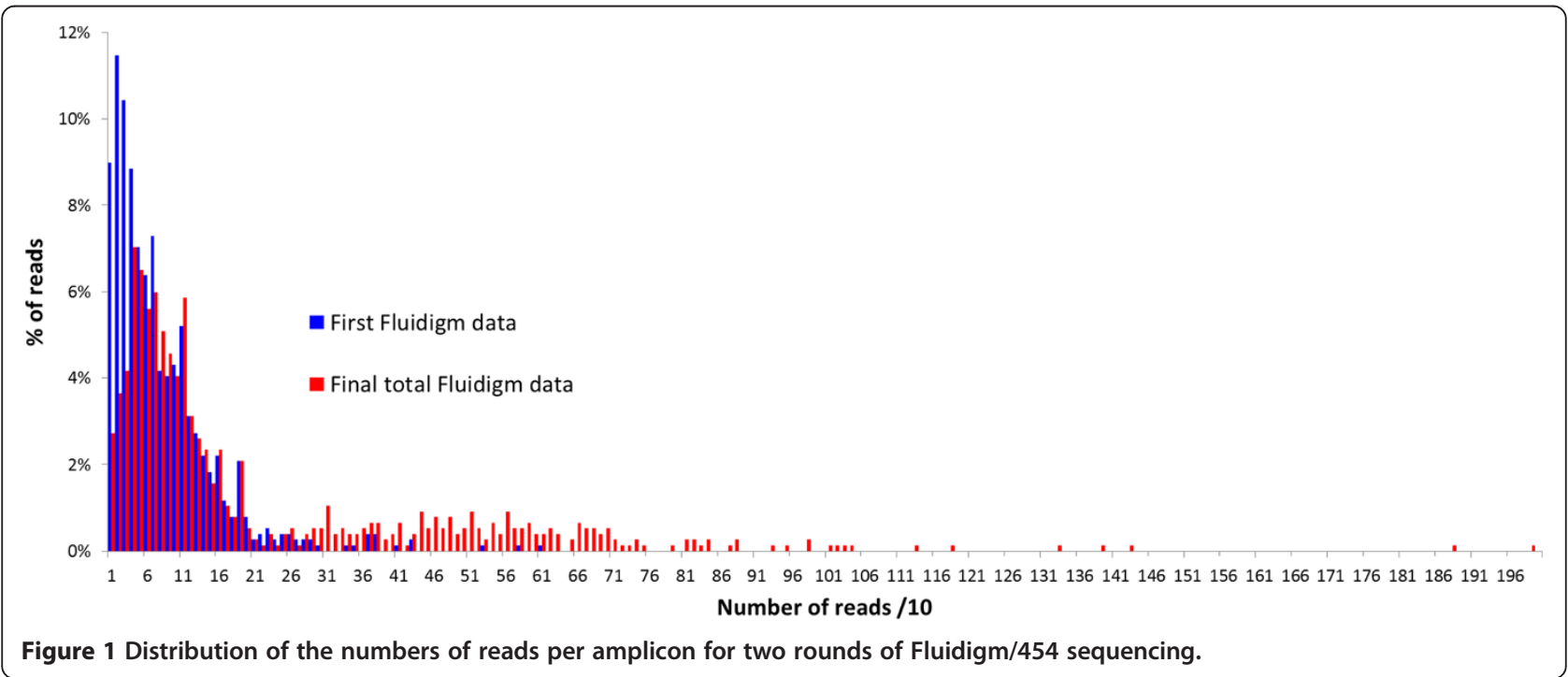


impact of the different SNPs according to the whole genome annotation available at phytozome.net. The vast majority (98\%) of the SNP loci was diallelic, but $2 \%$ (seven loci) were triallelic (Table 2). The tri-allelism was validated by Sanger sequencing (data not shown). Sanger sequencing of the 2P33506778 fragment was performed for 32 Citrus varieties to estimate the 454 SNP-calling error rate. Only three differences between 454 and Sanger data were observed over 17,152 bp genotyping data (32 genotypes per 536 bp fragment; $0.02 \%$ error rate). The 'Clemenules' clementine was homozygous according to Sanger sequencing, but had two heterozygotic SNPs according to the 454 sequencing data. The 'Beauty' mandarin was shown to be heterozygous with the two techniques, but one of the three heterozygotic 454 SNPs was not identified in the Sanger data. The average SNP frequencies in intronic, exonic and 3' UTR regions were 53.57, 38.77, and 39.77 $\mathrm{SNPs} / \mathrm{kb}$, respectively. In addition, five indels were found in exonic regions (fragments 2P8108334, 2P26819388, and 2P32507721 contained one indel, and 2P29538734 contained two).

\section{SNP diversity differentiation}

Previous molecular studies $[14,16,43]$ showed that some varieties of the main Citrus cultivar groups had interspecific introgressions. Therefore, in this study, we differentiated mandarin, pummelo, and citron groups of their respective pure ancestral taxa: $C$. reticulata, $C$. maxima, and C. medica.
For genotypic based analyses, we refer to the modern varietal groups, while we focus on pure ancestral taxa for the haplotype phylogenetic analyses.

Only 19 of the 318 SNPs were not found in the accessions representing the four basic taxa. These rare alleles were identified in heterozygosity in secondary species ('Alemow', nine; sour oranges, four; bergamot, three, 'Volkamer' lemon, one; 'Mexican' lime, one; and grapefruit, one) and concerned 9 of the 16 gene fragments. The parameters of SNP genetic diversity given in Table 3 (and detailed in [Additional file 6] for each SNP position) were calculated without these 19 rare alleles. The whole population displayed a diversity index $(\mathrm{He})$ of 0.23 and a fixation index $\left(F_{W}\right)$ value of 0.29 , suggesting an important population genetic structure of the analysed varietal sample. Mandarin and pummelo intra-diversity $\mathrm{F}_{\mathrm{W}}$ values were close to zero, but intra-group polymorphism was higher in mandarin $(\mathrm{He}=0.12 \pm 0.02)$ than in pummelo $(\mathrm{He}=0.07 \pm 0.02)$. Citron displayed low heterozygosity $(\mathrm{Ho}=0.02 \pm 0.01)$ and diversity $(\mathrm{He}=0.03 \pm 0.01)$. Only one $C$. micrantha representative was available: the observed heterozygosity value $(0.09 ; \pm 0.09)$ was, therefore, calculated between the pummelo and mandarin values. The average numbers of SNPs/kb between two varieties within and between the four supposed basic taxa were 1.26-3.93 SNPs/kb within groups and 10.41-14.56 $\mathrm{SNPs} / \mathrm{kb}$ at the inter-group level (Table 4).

For secondary species, no intraspecific polymorphism was observed for sweet oranges, grapefruits, and sour

Table 2 SNP number and location for 16 gene fragments sequenced in 48 diploid Citrus genotypes

\begin{tabular}{|c|c|c|c|c|c|c|c|c|c|c|c|c|c|}
\hline \multirow{2}{*}{$\begin{array}{l}\text { Gene } \\
\text { fragment }\end{array}$} & \multicolumn{4}{|c|}{ Total Sequence } & \multicolumn{3}{|l|}{ Intron } & \multicolumn{3}{|l|}{ Exon } & \multicolumn{3}{|l|}{ 3'-UTR } \\
\hline & Seq size & SNP & $\mathrm{SNP} / \mathbf{k b}$ & Trialelic SNP & Seq size & SNP & $\mathrm{SNP} / \mathrm{kb}$ & Seq size & SNP & $\mathrm{SNP} / \mathrm{kb}$ & Seq size & SNP & $\mathrm{SNP} / \mathbf{k b}$ \\
\hline 2P737170 & 452 & 22 & 48.67 & Exon & 0 & 0 & - & 452 & 22 & 48.67 & 0 & 0 & - \\
\hline 2P3068140 & 421 & 14 & 33.25 & - & 337 & 12 & 35.61 & 84 & 2 & 23.81 & 0 & 0 & - \\
\hline 2P4517048 & 502 & 12 & 23.90 & - & 0 & 0 & - & 316 & 4 & 12.66 & 186 & 8 & 43.01 \\
\hline 2P8108334 & 502 & 40 & 79.68 & Exon & 0 & 0 & - & 502 & 40 & 79.68 & 0 & 0 & - \\
\hline 2P11442721 & 547 & 21 & 38.39 & Exon & 0 & 0 & - & 547 & 21 & 38.39 & 0 & 0 & - \\
\hline 2P13928427 & 502 & 21 & 41.83 & - & 0 & 0 & - & 336 & 15 & 44.64 & 166 & 6 & 36.14 \\
\hline 2P21022460 & 538 & 11 & 20.45 & - & 0 & 0 & - & 538 & 11 & 20.45 & 0 & 0 & - \\
\hline 2P25198627 & 454 & 12 & 26.43 & - & 128 & 7 & 54.69 & 326 & 5 & 15.34 & 0 & 0 & - \\
\hline 2P26819388 & 535 & 22 & 41.12 & Exon & 0 & 0 & - & 535 & 22 & 41.12 & 0 & 0 & - \\
\hline 2P29538734 & 541 & 36 & 66.54 & Exon & 190 & 12 & 63.16 & 351 & 24 & 68.38 & 0 & 0 & - \\
\hline 2P30446231 & 475 & 28 & 58.95 & - & 216 & 15 & 69.44 & 259 & 13 & 50.19 & 0 & 0 & - \\
\hline 2P32507721 & 463 & 16 & 34.56 & - & 0 & 0 & - & 463 & 16 & 34.56 & 0 & 0 & - \\
\hline 2P33532337 & 459 & 9 & 19.61 & - & 0 & 0 & - & 459 & 9 & 19.61 & 0 & 0 & - \\
\hline 2P33506778 & 536 & 6 & 11.19 & Exon & 0 & 0 & - & 536 & 6 & 11.19 & 0 & 0 & - \\
\hline 2P35391362 & 449 & 19 & 42.32 & - & 108 & 6 & 55.56 & 341 & 13 & 38.12 & 0 & 0 & - \\
\hline 2P36235952 & 519 & 29 & 55.88 & Exon & 141 & 8 & 56.74 & 378 & 21 & 55.56 & 0 & 0 & - \\
\hline 16 & 7895 & 318 & 40.28 & & 1120 & 60 & 53.57 & 6423 & 244 & 37.99 & 352 & 14 & 39.77 \\
\hline
\end{tabular}


Table 3 SNP genetic diversity within and between supposed ancestral varietal groups

\begin{tabular}{|c|c|c|c|c|c|c|c|c|c|c|c|c|c|c|c|c|}
\hline & \multicolumn{3}{|c|}{ Whole population } & \multicolumn{3}{|c|}{ Citrons } & \multicolumn{3}{|c|}{ Mandarins } & \multicolumn{3}{|c|}{ Citrus micrantha } & \multicolumn{3}{|c|}{ Pummelos } & \multirow{2}{*}{$\begin{array}{l}4 \text { population } \\
F_{S T}\end{array}$} \\
\hline & Ho & $\mathrm{He}$ & $F_{W}$ & Ho & $\mathrm{He}$ & $\mathrm{F}_{\mathrm{W}}$ & Ho & $\mathrm{He}$ & $F_{W}$ & Ho & $\mathrm{He}$ & $F_{W}$ & Ho & $\mathrm{He}$ & $\mathrm{F}_{\mathrm{W}}$ & \\
\hline 2P737170 & 0.11 & 0.23 & 0.52 & 0.02 & 0.03 & 0.33 & 0.01 & 0.01 & -0.04 & 0.15 & 0.08 & -1.00 & 0.08 & 0.07 & -0.22 & 0.78 \\
\hline SD & 0.07 & 0.15 & 0.25 & 0.02 & 0.11 & 0.00 & 0.03 & 0.02 & 0.00 & 0.36 & 0.18 & 0.00 & 0.04 & 0.15 & 0.08 & 0.33 \\
\hline $\mathrm{Cl}$ & 0.03 & 0.07 & 0.11 & 0.02 & 0.05 & - & 0.01 & 0.01 & - & 0.16 & 0.08 & - & 0.02 & 0.07 & 0.08 & 0.14 \\
\hline 2P3068140 & 0.18 & 0.33 & 0.46 & 0.00 & 0.00 & - & 0.07 & 0.10 & -0.01 & 0.00 & 0.00 & - & 0.02 & 0.02 & -0.18 & 0.72 \\
\hline SD & 0.08 & 0.17 & 0.22 & 0.00 & 0.00 & - & 0.12 & 0.10 & 0.13 & 0.00 & 0.00 & - & 0.04 & 0.07 & - & 0.40 \\
\hline $\mathrm{Cl}$ & 0.05 & 0.09 & 0.12 & - & - & - & 0.06 & 0.05 & 0.07 & - & - & - & 0.02 & 0.04 & - & 0.22 \\
\hline 2P4517048 & 0.09 & 0.19 & 0.55 & 0.01 & 0.01 & -0.09 & 0.10 & 0.08 & -0.17 & 0.00 & 0.00 & - & 0.06 & 0.05 & -0.14 & 0.48 \\
\hline SD & 0.07 & 0.19 & 0.33 & 0.03 & 0.04 & - & 0.07 & 0.18 & 0.22 & 0.00 & 0.00 & - & 0.08 & 0.09 & 0.04 & 0.47 \\
\hline $\mathrm{Cl}$ & 0.04 & 0.11 & 0.19 & 0.02 & 0.02 & - & 0.04 & 0.10 & 0.25 & - & - & - & 0.05 & 0.05 & 0.04 & 0.27 \\
\hline 2P8108334 & 0.12 & 0.20 & 0.42 & 0.01 & 0.04 & 0.80 & 0.04 & 0.06 & 0.38 & 0.21 & 0.10 & -1.00 & 0.14 & 0.09 & -0.52 & 0.52 \\
\hline SD & 0.09 & 0.18 & 0.36 & 0.01 & 0.10 & 0.49 & 0.05 & 0.14 & 0.28 & 0.41 & 0.20 & 0.00 & 0.06 & 0.17 & 0.33 & 0.36 \\
\hline $\mathrm{Cl}$ & 0.03 & 0.06 & 0.11 & 0.01 & 0.03 & 0.39 & 0.03 & 0.04 & 0.18 & 0.13 & 0.06 & - & 0.04 & 0.05 & 0.20 & 0.11 \\
\hline 2P11442721 & 0.15 & 0.18 & 0.18 & 0.02 & 0.02 & -0.09 & 0.23 & 0.20 & -0.19 & 0.00 & 0.00 & - & 0.06 & 0.06 & 0.04 & 0.32 \\
\hline SD & 0.11 & 0.13 & 0.26 & 0.05 & 0.05 & 0.00 & 0.09 & 0.20 & 0.16 & 0.00 & 0.00 & - & 0.06 & 0.12 & 0.32 & 0.35 \\
\hline $\mathrm{Cl}$ & 0.05 & 0.05 & 0.11 & 0.04 & 0.02 & 0.00 & 0.05 & 0.08 & 0.09 & - & - & - & 0.04 & 0.05 & 0.28 & 0.15 \\
\hline 2P13928427 & 0.11 & 0.17 & 0.38 & 0.04 & 0.05 & 0.17 & 0.06 & 0.05 & -0.11 & 0.10 & 0.05 & -1.00 & 0.01 & 0.01 & -0.05 & 0.40 \\
\hline SD & 0.08 & 0.16 & 0.23 & 0.04 & 0.15 & 0.00 & 0.11 & 0.08 & 0.05 & 0.30 & 0.15 & 0.00 & 0.03 & 0.03 & 0.00 & 0.38 \\
\hline $\mathrm{Cl}$ & 0.04 & 0.07 & 0.10 & 0.04 & 0.06 & - & 0.06 & 0.04 & 0.04 & 0.13 & 0.07 & - & 0.02 & 0.01 & - & 0.16 \\
\hline 2P21022460 & 0.12 & 0.19 & 0.34 & 0.00 & 0.04 & 1.00 & 0.05 & 0.06 & 0.11 & 0.18 & 0.09 & -1.00 & 0.10 & 0.07 & -0.50 & 0.49 \\
\hline SD & 0.08 & 0.13 & 0.28 & 0.00 & 0.13 & - & 0.07 & 0.13 & 0.11 & 0.39 & 0.20 & 0.00 & 0.03 & 0.16 & 0.35 & 0.41 \\
\hline $\mathrm{Cl}$ & 0.05 & 0.08 & 0.16 & - & 0.08 & - & 0.03 & 0.08 & 0.11 & 0.23 & 0.12 & - & 0.02 & 0.09 & 0.48 & 0.24 \\
\hline 2P25198627 & 0.17 & 0.21 & 0.20 & 0.08 & 0.10 & 0.16 & 0.10 & 0.13 & 0.23 & 0.17 & 0.08 & -1.00 & 0.20 & 0.15 & -0.38 & 0.34 \\
\hline SD & 0.14 & 0.16 & 0.20 & 0.08 & 0.18 & 0.61 & 0.14 & 0.18 & 0.22 & 0.37 & 0.19 & 0.00 & 0.10 & 0.22 & 0.05 & 0.33 \\
\hline $\mathrm{Cl}$ & 0.08 & 0.09 & 0.12 & 0.06 & 0.10 & 0.69 & 0.07 & 0.10 & 0.15 & 0.21 & 0.11 & - & 0.06 & 0.12 & 0.05 & 0.19 \\
\hline 2P26819388 & 0.09 & 0.16 & 0.46 & 0.07 & 0.05 & -0.33 & 0.08 & 0.13 & 0.45 & 0.32 & 0.17 & -1.00 & 0.02 & 0.02 & -0.33 & 0.25 \\
\hline SD & 0.10 & 0.17 & 0.30 & 0.06 & 0.13 & 0.00 & 0.10 & 0.19 & 0.36 & 0.47 & 0.24 & 0.00 & 0.02 & 0.08 & - & 0.18 \\
\hline $\mathrm{Cl}$ & 0.04 & 0.07 & 0.12 & 0.05 & 0.05 & - & 0.07 & 0.08 & 0.21 & 0.19 & 0.10 & - & 0.01 & 0.03 & - & 0.07 \\
\hline 2P29538734 & 0.17 & 0.26 & 0.34 & 0.00 & 0.03 & 1.00 & 0.19 & 0.16 & -0.18 & 0.06 & 0.03 & -1.00 & 0.05 & 0.05 & -0.07 & 0.53 \\
\hline SD & 0.11 & 0.16 & 0.28 & 0.00 & 0.09 & 0.00 & 0.16 & 0.17 & 0.09 & 0.23 & 0.11 & 0.00 & 0.06 & 0.10 & 0.37 & 0.39 \\
\hline $\mathrm{Cl}$ & 0.04 & 0.05 & 0.09 & - & 0.03 & - & 0.08 & 0.05 & 0.04 & 0.08 & 0.04 & - & 0.04 & 0.03 & 0.23 & 0.13 \\
\hline 2Р30446231 & 0.12 & 0.20 & 0.37 & 0.02 & 0.04 & 0.56 & 0.10 & 0.11 & 0.07 & 0.08 & 0.04 & -1.00 & 0.10 & 0.16 & 0.39 & 0.47 \\
\hline SD & 0.09 & 0.14 & 0.28 & 0.04 & 0.12 & 0.00 & 0.12 & 0.17 & 0.11 & 0.27 & 0.14 & 0.00 & 0.07 & 0.20 & 0.44 & 0.37 \\
\hline $\mathrm{Cl}$ & 0.03 & 0.05 & 0.11 & 0.03 & 0.05 & - & 0.06 & 0.07 & 0.07 & 0.10 & 0.05 & - & 0.05 & 0.08 & 0.26 & 0.14 \\
\hline 2P32507721 & 0.14 & 0.21 & 0.31 & 0.00 & 0.00 & - & 0.29 & 0.31 & 0.06 & 0.18 & 0.08 & -1.00 & 0.07 & 0.04 & -0.60 & 0.43 \\
\hline SD & 0.10 & 0.14 & 0.18 & 0.00 & 0.00 & - & 0.25 & 0.24 & 0.05 & 0.39 & 0.19 & 0 & 0.04 & 0.14 & - & 0.32 \\
\hline $\mathrm{Cl}$ & 0.06 & 0.08 & 0.10 & - & - & - & 0.13 & 0.14 & 0.03 & 0.23 & 0.11 & - & 0.02 & 0.08 & - & 0.18 \\
\hline 2P33506778 & 0.18 & 0.29 & 0.37 & 0.00 & 0.00 & - & 0.18 & 0.17 & -0.07 & 0.00 & 0.00 & - & 0.05 & 0.06 & 0.20 & 0.81 \\
\hline SD & 0.13 & 0.17 & 0.11 & 0.00 & 0.00 & - & 0.17 & 0.21 & 0.02 & 0.00 & 0.00 & - & 0.08 & 0.15 & - & 0.20 \\
\hline $\mathrm{Cl}$ & 0.10 & 0.13 & 0.09 & - & - & - & 0.09 & 0.17 & 0.03 & - & - & - & 0.05 & 0.12 & - & 0.16 \\
\hline 2P33532337 & 0.19 & 0.32 & 0.39 & 0.00 & 0.07 & 1.00 & 0.23 & 0.24 & -0.14 & 0.00 & 0.00 & - & 0.04 & 0.09 & 0.52 & 0.65 \\
\hline SD & 0.11 & 0.15 & 0.15 & 0.00 & 0.08 & 0.00 & 0.26 & 0.17 & 0.20 & 0.00 & 0.00 & - & 0.09 & 0.19 & 0.00 & 0.31 \\
\hline $\mathrm{Cl}$ & 0.07 & 0.10 & 0.10 & - & 0.05 & - & 0.14 & 0.11 & 0.15 & - & - & - & 0.06 & 0.12 & - & 0.21 \\
\hline
\end{tabular}


Table 3 SNP genetic diversity within and between supposed ancestral varietal groups (Continued)

\begin{tabular}{lllllllllllllllllll}
\hline 2P35391362 & 0.22 & 0.37 & 0.41 & 0.01 & 0.01 & -0.09 & 0.24 & 0.20 & -0.24 & 0.00 & 0.00 & - & 0.02 & 0.06 & -0.15 & 0.73 & \\
SD & 0.09 & 0.13 & 0.16 & 0.02 & 0.04 & - & 0.24 & 0.18 & 0.10 & 0.00 & 0.00 & - & 0.03 & 0.09 & 0.09 & 0.24 & \\
Cl & 0.04 & 0.06 & 0.07 & 0.02 & 0.02 & - & 0.13 & 0.08 & 0.06 & - & - & - & 0.02 & 0.04 & 0.06 & 0.11 & \\
2P36235952 & 0.13 & 0.28 & 0.55 & 0.00 & 0.00 & - & 0.02 & 0.04 & 0.45 & 0.04 & 0.02 & -1.00 & 0.08 & 0.07 & -0.28 & 0.55 & \\
SD & 0.08 & 0.21 & 0.34 & 0.00 & 0.00 & - & 0.04 & 0.11 & 0.35 & 0.20 & 0.10 & 0.00 & 0.02 & 0.13 & 0.15 & 0.47 & 0.13 \\
Cl & 0.03 & 0.08 & 0.14 & - & - & - & 0.02 & 0.04 & 0.26 & 0.08 & 0.04 & - & 0.01 & 0.05 & 0.12 & 0.19 & \\
Total & 0.14 & 0.23 & 0.29 & 0.02 & 0.03 & 0.46 & 0.12 & 0.12 & -0.02 & 0.09 & 0.05 & -1.00 & 0.07 & 0.07 & -0.08 & 0.51 & \\
SD & 0.10 & 0.17 & 0.28 & 0.01 & 0.10 & 0.53 & 0.05 & 0.17 & 0.24 & 0.19 & 0.15 & 0.00 & 0.02 & 0.14 & 0.37 & 0.38 & \\
Cl & 0.03 & 0.02 & 0.03 & 0.01 & 0.01 & 0.19 & 0.03 & 0.02 & 0.04 & 0.09 & 0.02 & - & 0.01 & 0.02 & 0.09 & 0.04 & \\
\hline
\end{tabular}

Ho: observed heterozygosity; He: expected heterozygosity; $\mathrm{F}_{\mathrm{W}}$ : fixation index; $\mathrm{F}_{\mathrm{ST}}$ : fixation index within population; SD: standard deviation; Cl: confidence interval estimated with alpha $=0.05$.

oranges, represented, respectively, by three, two, and two varieties. The two clementine cultivars were also found to be identical. Polymorphism was found between regular lemons and the other ones; however, the two regular lemons ('Eureka' and 'Lisbon') and 'Sweet' lemon were found to be identical. Acid citrus types (lemons, limes, 'Alemow', and bergamot) and sour orange displayed high Ho values $(0.26-0.34 \pm 0.05)$. Sweet orange $(0.15 \pm 0.04)$, clementine $(0.19 \pm 0.04)$, and grapefruit $(0.12 \pm 0.04)$ displayed comparatively lower heterozygosity levels [Additional file 7].

Structure software analysis was performed in the absence of a prior hypothesis for group number. Analysis of $\Delta \mathrm{K}$ identified $\mathrm{K}=4$ as the optimal population number. The ten runs for $K=4$ displayed very homogeneous results (as shown by the average values [Figure 2, Additional file 8]). C. medica, C. maxima, and C. micrantha defined three populations, and five mandarins defined a fourth population. The magnitude of genetic differentiation between the groups was statistically confirmed by the pairwise $F_{\mathrm{ST}}$ values, which ranged from $0.499 \pm 0.091$ for C. maxima/C. micrantha to $0.719 \pm 0.087$ for $C$. micrantha/C. medica (Table 5). Eight of the additional mandarins appeared to belong chiefly to this last group but exhibited introgression from the $C$. maxima group. 'Shekwasha' mandarin displayed a possible introgression of C. micrantha. Some cultivars displayed more

Table 4 Intra- and inter-varietal group dissimilarities (average number of SNP/kb between two varieties)

\begin{tabular}{llll}
\hline & Mandarins & Pummelos & Citrons \\
\hline Mandarins & $3.93^{*}$ & & \\
Pummelos & 10.41 & $2.06^{*}$ & \\
Citrons & 14.56 & 11.21 & $1.26^{*}$ \\
C. micrantha & 13.49 & 10.61 & 12.24 \\
\hline
\end{tabular}

*Average number of SNP/kb at intra-specific level. pronounced genetic mixing. 'Alemow' and 'Mexican' lime had half their features from the C. micrantha group and half from the $C$. medica group. Similarly, sour oranges had half their features from each of the $C$. reticulata and $C$. maxima groups. Sweet orange and clementine were admixtures of the C. maxima and C. reticulata groups.. Regular and 'Sweet' lemons and bergamot were admixtures of three groups: C. maxima, C. reticulata, and $C$. medica. Close to half of the genetic material in 'Volkamer' and 'Meyer' lemons was of the C. medica group, and half was of the $C$. reticulata group [Figure 2, Additional file 8].

PCA analysis confirmed the organization of the whole diversity coming from the four ancestral varietal groups (Figure 3). The three primary axes encompassed $56.3 \%$ of the total observed diversity. The first axis mainly separated citrons and C. micrantha from pummelos and mandarins. The second axis distinguished pummelos from other ancestral varietal groups. Finally, the third axis separated C. micrantha from other groups. 'Alemow' and 'Mexican' lime displayed intermediate positions between citrons and C. micrantha. Regular and 'Sweet' lemons and bergamot had intermediate positions between citrons and mandarins/sour oranges. Clementine lay within the mandarin cluster, while grapefruit was included in the pummelo cluster. Sweet orange and sour orange were located between the pummelo and mandarin clusters. The mandarin group displayed two noticeable subclusters. The subcluster that contained clementines and mandarins that were potentially introgressed by pummelo was displaced towards the pummelo cluster.

Analysis of linkage disequilibrium (LD) between SNPs along the chromosome [Additional file 9] also testifieds to a very high population genetic structure of the varietal sample. Significant LD values were observed across the whole chromosome, even for SNPs at distally opposing positions. 


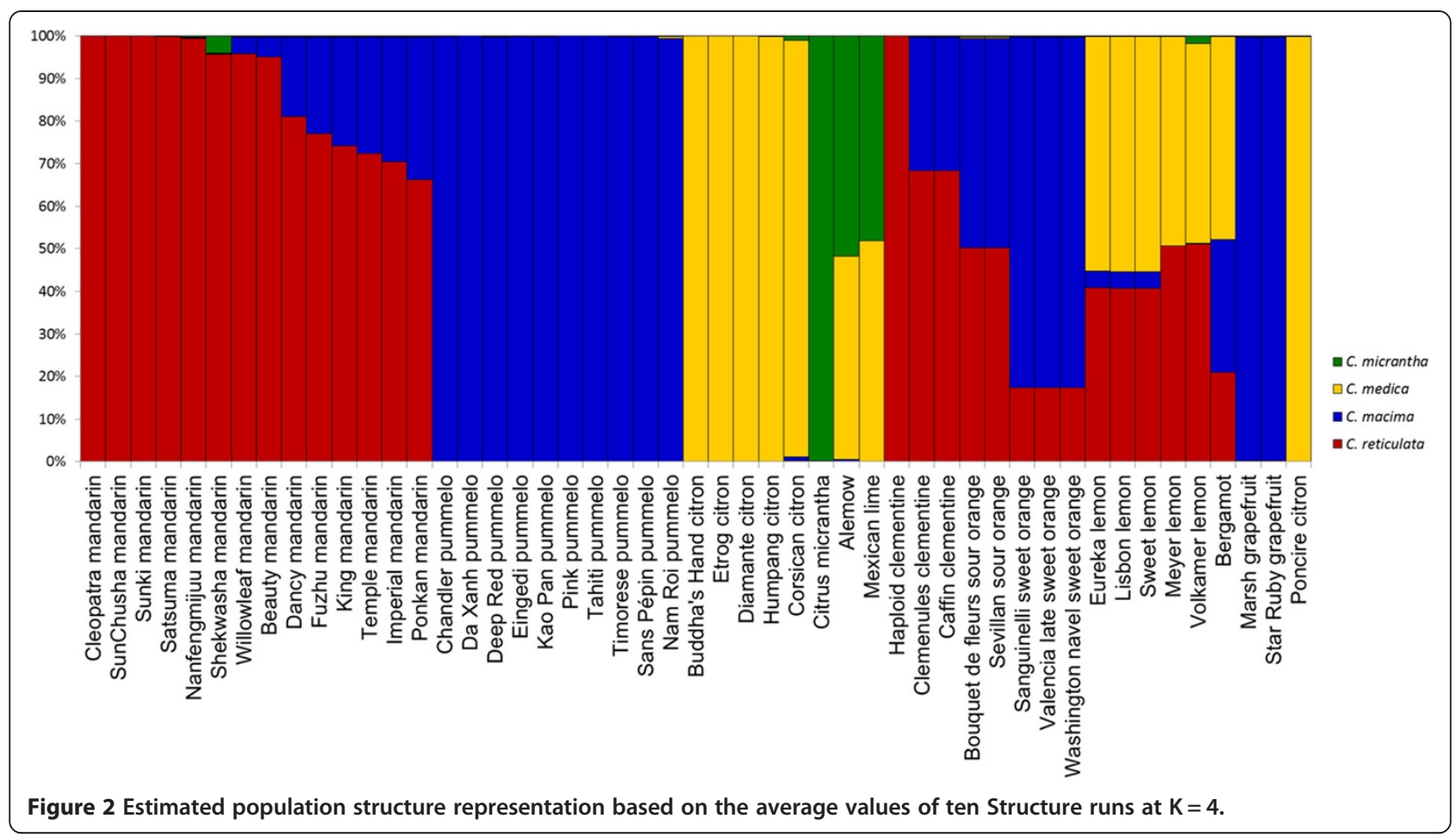

\section{Gene fragment haplotype inference and phylogeny}

For each gene fragment, two haplotypes were inferred for each variety. NJA and maximum likelihood analysis of haplotypes was performed to determine phylogenetic relationships, and the two methods produced the same outcomes. For example, for the 2P35391362 gene fragment (Figures 4), three, three, one, and two different haplotypes were identified in the $C$. reticulata, C. maxima, C. micrantha, and C. medica clusters, respectively. Multilocus haplotypic analysis also provided evidence of interspecific introgressions in varieties representative of one of the four supposed ancestral varietal groups. For this fragment, six mandarins shared one $C$. maxima haplotype with pummelos. Haplotypic analysis allowed clear inference of phylogenetic inheritance patterns for 2P35391362 in the secondary citrus species [Additional file 10]. For example, clementine clearly exhibited interspecific heterozygosity (C. maxima/C. reticulata): one haplotype was shared with sweet orange in the C. maxima cluster, and one was shared with 'Willowleaf' mandarin in the $C$. reticulata cluster. The second sweet orange haplotype was also in the C. maxima cluster and was shared with grapefruits that were homozygous for this haplotype. Evidence of interspecific inheritance was also found in sour orange (C. maxima/C. reticulata), bergamot (C. medica/C. reticulata), 'Eureka, 'Lisbon, 'Sweet', 'Volkamer', and 'Meyer' lemons (C. medica/C. reticulata), and 'Mexican' lime and 'Alemow' (C. medica/C. micrantha). NJA of genotypic information from the same data set (Figure 5) provided a representation of two apparent $C$. reticulata clusters with unclear relationships. One of the clusters included accessions that exhibited interspecific inheritance when haplotype was assessed (several mandarins, sour oranges, and clementines). Similarly, lemons, limes, 'Alemow', and bergamot lay between $C$. medica and $C$. micrantha, clusters and branching did not provide definitive phylogenetic information.

A total of 210 haplotypes were identified through analysis of 16 gene fragments on chromosome 2 (Table 6; [Additional file 11]). From the phylogenetic analysis

Table 5 Pairwise population $\mathrm{F}_{\mathrm{ST}}$ values

\begin{tabular}{|c|c|c|c|c|c|c|}
\hline & \multicolumn{2}{|c|}{ Mandarin } & \multicolumn{2}{|c|}{ Pummelo } & \multicolumn{2}{|c|}{ Citron } \\
\hline & SNP & $\mathrm{F}_{\mathrm{ST}}$ & SNP & $\mathrm{F}_{\mathrm{ST}}$ & SNP & $\mathrm{F}_{\mathrm{ST}}$ \\
\hline Pummelo & 172 & $0.502 \pm 0.061$ & & & & \\
\hline Citron & 171 & $0.666 \pm 0.061$ & 142 & $0.585 \pm 0.066$ & & \\
\hline C. micrantha & 167 & $0.574 \pm 0.079$ & 143 & $0.499 \pm 0.091$ & 127 & $0.719 \pm 0.087$ \\
\hline
\end{tabular}




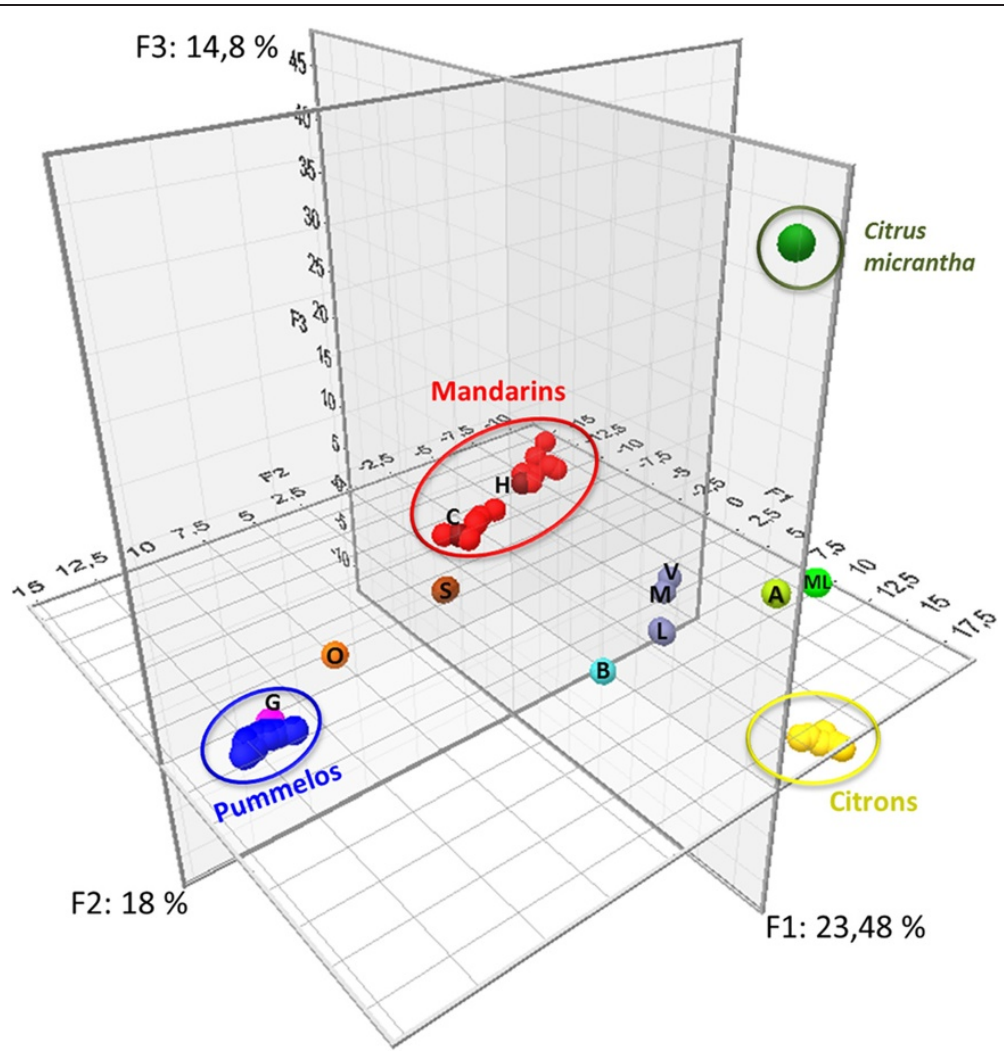

Figure 3 Organization of genotypic SNP diversity. All varieties and all SNP data were analyzed by PCA. ML: 'Mexican' lime; A: 'Alemow'; V: Volkamer' lemon; M: 'Meyer' lemon; L: Regular and 'Sweet' lemons; B: Bergamot; H: Haploid clementine; C: Clementines; S: Sour oranges; O: Sweet oranges; G: Grapefruits.

of each fragment, we considered $77,58,34$, and 25 haplotypes to be representative of $C$. reticulata, $C$. maxima, C. medica, and C. micrantha, respectively. For 16 haplotypes, the organization of the genetic diversity of the corresponding fragment was insufficient to infer phylogenetic origin. The indeterminate haplotypes mostly concerned mandarin and pummelo.

The haplotypic structure of each accession was used to schematize the phylogenetic origin of genome fragments along chromosome 2 (Figure 6). In the absence of data regarding the phase between different haplotypes, this representation was made genotypically (homozygous for one ancestral taxon or heterozygous between two taxa). A single genotype was used to represent a varietal group when no polymorphisms were observed between varieties.

Ten of the fourteen mandarins were introgressed by C. maxima, mostly in heterozygosity. Two homozygous fragments for a C. maxima haplotype (ma1/ma1) and one fragment heterozygous for two $C$. maxima haplotypes (ma1/ma2) were found in 'Ponkan' mandarin. No evidence of interspecific introgression was observed for the representatives of the other three ancestral varietal groups.
Completely heterozygous interspecific structures between C. micrantha and C. medica were observed for 'Mexican' lime and 'Alemow'. Sour orange displayed complete heterozygosity between $C$. reticulata and C. maxima. Grapefruit appeared to have inherited mostly C. maxima haplotypes but displayed heterozygosity with $C$. reticulata at the start of the scaffold. Sweet orange was mostly heterozygous between $C$. reticulata and C. maxima, with a small fragment at the first part of the scaffold inherited solely from C. reticulata, and a genome area at the end of the scaffold inherited exclusively from C. maxima. Bergamot and regular, 'Sweet', and 'Meyer' lemons displayed similar structures that mainly comprised heterozygous regions of C. medica/C. reticulata and C. medica/C. maxima. However, two small homozygous regions (2P4517048 and 2P33532337 gene fragments) were observed in 'Meyer' lemon (C. reticulata homozygosity re3/re3 and re2/re2) and bergamot (C. maxima homozygosity ma1/ma1 and $\mathrm{ma} 2 / \mathrm{ma} 2)$. No exploitable data were obtained for one gene fragment of 'Volkamer' lemon. For the other 15 gene fragments, 'Volkamer' lemon systematically displayed one haplotype corresponding with the $C$. medica cluster. The other haplotypes for 14 of these gene fragments 


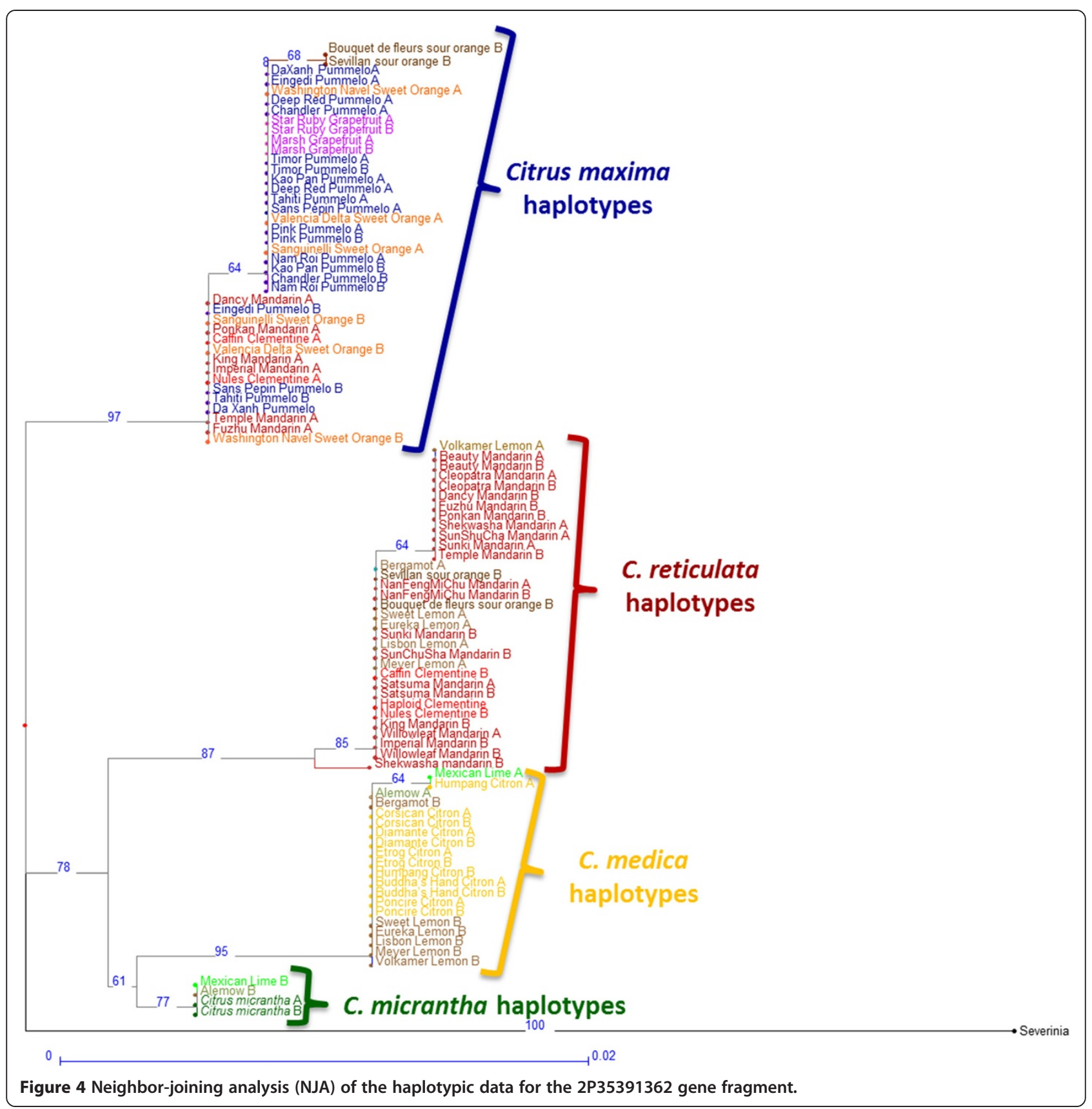

were assigned to the $C$. reticulata cluster. The remaining haplotype was in a cluster of indeterminate phylogeny [Additional file 12].

Revised genetic relationships between the four basic taxa after removal of introgressed genomic regions identified in mandarin from haplotypic analysis

The identification, from haplotypic analysis, of introgressed pummelo fragments in mandarin genotypes prompted a revision of the relationships of the ancestral basic taxa (C. maxima, C. reticulata, C. medica, and C. micrantha) relative to the varietal groups deriving from these taxa (pummelos, mandarins, citrons and micrantha). The average SNP density within $C$. reticulata (Table 7) was lower $(2.85 \mathrm{SNP} / \mathrm{kb})$ than in mandarin $(3.93 \mathrm{SNP} / \mathrm{kb})$ (Table 4). Conversely, the $C$. maxima/C. reticulata average differentiation was $11.15 \mathrm{SNP} / \mathrm{kb}$ (10.41 SNP/kb between mandarins and pummelos). The differentiation values of $C$. reticulata with $C$. micrantha and $C$. medica were similar to those of mandarin with micrantha and citron, respectively.

For each SNP, $\mathrm{G}_{\mathrm{ST}}$ values were estimated for each basic species relative to all other species. This allowed estimation of the value of each considered SNP to confirm that 


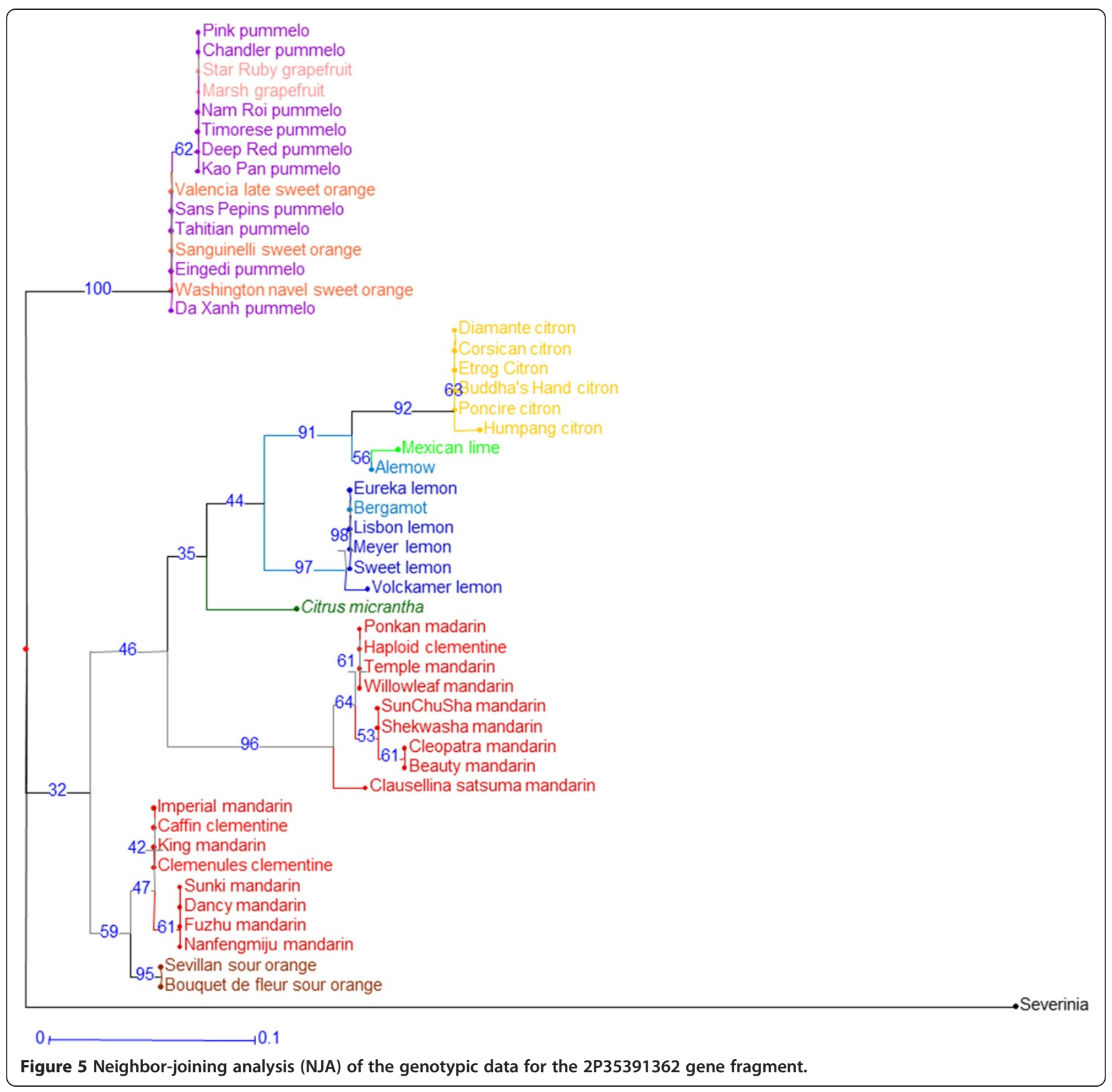

the surrounding genome fragment was inherited from the given species (SNP specific-diagnostic points). Corrections from the introgression information increased the number of diagnostic markers for C. reticulata and C. maxima relative to the initial data for mandarin and pummelo [Additional file 13]. The number of SNP loci with an average $\mathrm{G}_{\mathrm{ST}}$ value $>0.8$ increased from 14 and 6 for mandarins and pummelos to 27 and 10 for C. reticulata and C. max$i m a$, respectively. The highest number of totally discriminant SNPs $\left(\mathrm{G}_{\mathrm{ST}}=1\right)$ was observed for C. medica (27) followed by C. reticulata (22), C. micrantha (21), and $C$. maxima (8) [Additional file 14].

\section{Discussion}

Genotype and haplotype information from 454 parallel sequencing of 400-600 bp amplicons can identify admixture structures and infer the evolutionary history of species with reticulate evolution

Three hundred heighten SNPs were found in 16 gene fragments from chromosome 2 . The $\mathrm{SNPs} / \mathrm{kb}$ rate within introns (53.6) was highly similar to the rate previously determined for the Citrus genus (51.5) by Garcia-Lor et al. [16]. The SNPs/kb rate within exons was slightly higher in this study (38.0) than in the previous study (29). Taken together, and including the small 3' UTR regions, 48.3 
Table 6 Number of haplotypes attributed to the four basic taxa or with indeterminate phylogenetic origin

\begin{tabular}{|c|c|c|c|c|c|c|}
\hline Gene fragment & C. reticulata & C. maxima & C. medica & C. micrantha & Indeterminate & Total \\
\hline 2P737170 & 4 & 6 & 2 & 2 & 0 & 14 \\
\hline 2P3068140 & 4 & 2 & 1 & 1 & 1 & 9 \\
\hline 2P4517048 & 5 & 3 & 2 & 1 & 0 & 11 \\
\hline 2P8108334 & 10 & 7 & 3 & 2 & 2 & 24 \\
\hline 2P11442721 & 8 & 5 & 2 & 1 & 0 & 16 \\
\hline 2P13928427 & 3 & 2 & 2 & 2 & 2 & 11 \\
\hline 2P21022460 & 1 & 2 & 2 & 1 & 2 & 8 \\
\hline 2P25198627 & 5 & 1 & 3 & 2 & 1 & 12 \\
\hline 2P26819388 & 8 & 2 & 2 & 1 & 2 & 15 \\
\hline 2P29538734 & 7 & 6 & 4 & 2 & 0 & 19 \\
\hline 2P30446231 & 6 & 7 & 3 & 2 & 1 & 19 \\
\hline 2P32507721 & 3 & 1 & 1 & 2 & 4 & 11 \\
\hline 2P33506778 & 2 & 1 & 1 & 1 & 1 & 6 \\
\hline 2P33532337 & 2 & 2 & 1 & 1 & 0 & 6 \\
\hline 2P35391362 & 3 & 3 & 2 & 1 & 0 & 9 \\
\hline 2P36235952 & 6 & 8 & 3 & 3 & 0 & 20 \\
\hline Total & 77 & 58 & 34 & 25 & 16 & 210 \\
\hline
\end{tabular}

SNPs/kb were identified. This rate varied between gene fragments (range: 11.2-79.7).

The observed higher heterozygosity in secondary species than in the basic taxa, as well as the higher diversity in mandarin and pummelo compared to citron, was in agreement with previous studies $[15,16,18]$. Moreover, the high structuration of the diversity around $C$. maxima, $C$. medica, $C$. reticulata, and C. micrantha revealed by Structure and PCA agreed with previous molecular $[13,14,16,65]$ and numerical taxonomy [20] studies, which recognizes the four basic taxa as the ancestors of the cultivated Citrus species. The important ancestral taxon differentiation and the limited number of reticulations and further interspecific hybridizations also resulted in the generalized LD observed in this study. LD was maintained even for fragments on opposing telomeres, also noted in previous studies for markers on different chromosomes [15,18].

The relative levels of differentiation between C. maxima, C. medica, C. reticulata, and C. micrantha varied (10.61-14.8 SNPs/kb), and was on average 6.7 times higher than the within-taxon diversity (from 1.24 in $C$. medica to 2.85 in C. reticulata). This diversity pattern allowed inferring haplotype phylogenetic origin for 12 of the 16 genes examined on chromosome 2. Differentiation was low for the four genes in the central part of the chromosome, and this resulted in clusters of indeterminate phylogenetic origin. The indeterminate haplotypes mainly concerned mandarins, pummelos, and their secondary species haplotypes.

Haplotype analysis demonstrated C. maxima introgressions in genotypes generally considered to be true mandarins. After removal of these haplotypes from the analysis of the supposed ancestral taxa, higher monolocus differentiation was observed between $C$. reticulata and $C$. maxima. This also allowed more precise estimations of $C$. reticulata intraspecific polymorphism. The identification of introgressed areas from haplotypic analysis, therefore, provided better species tree reconstruction. As recommended by Ramagudu et al. [37], species trees can be improved by using loci that generate gene trees that are more clearly resolved. Haplotypic analysis has potential in this regard, and will allow the deselection of regions with incomplete lineage sorting or interspecific introgressions.

In the present study, 454 amplicon sequencing was successfully used to determine haplotypes in heterozygous genotypes and to analyze admixtures resulting from reticulate evolution. The broader utility of this method for identifying polymorphisms and inferring haplotype phylogenetic origins in other plants will depend on polymorphism rates within and between subspecies or species.

\section{Determination of the phylogenetic structure of} chromosome 2 in several Citrus species and varieties provided insights into the origins of modern cultivated citrus

Haplotype NJA analysis of each gene fragment allowed the phylogenetic inheritance of genome fragments along chromosome 2 to be inferred for the 48 analyzed genotypes. Although a small number of haplotypes remained of indeterminate phylogenetic origin, the results provided an invaluable overview of the phylogenetic structure of chromosome 2 and the origin of modern Citrus. 


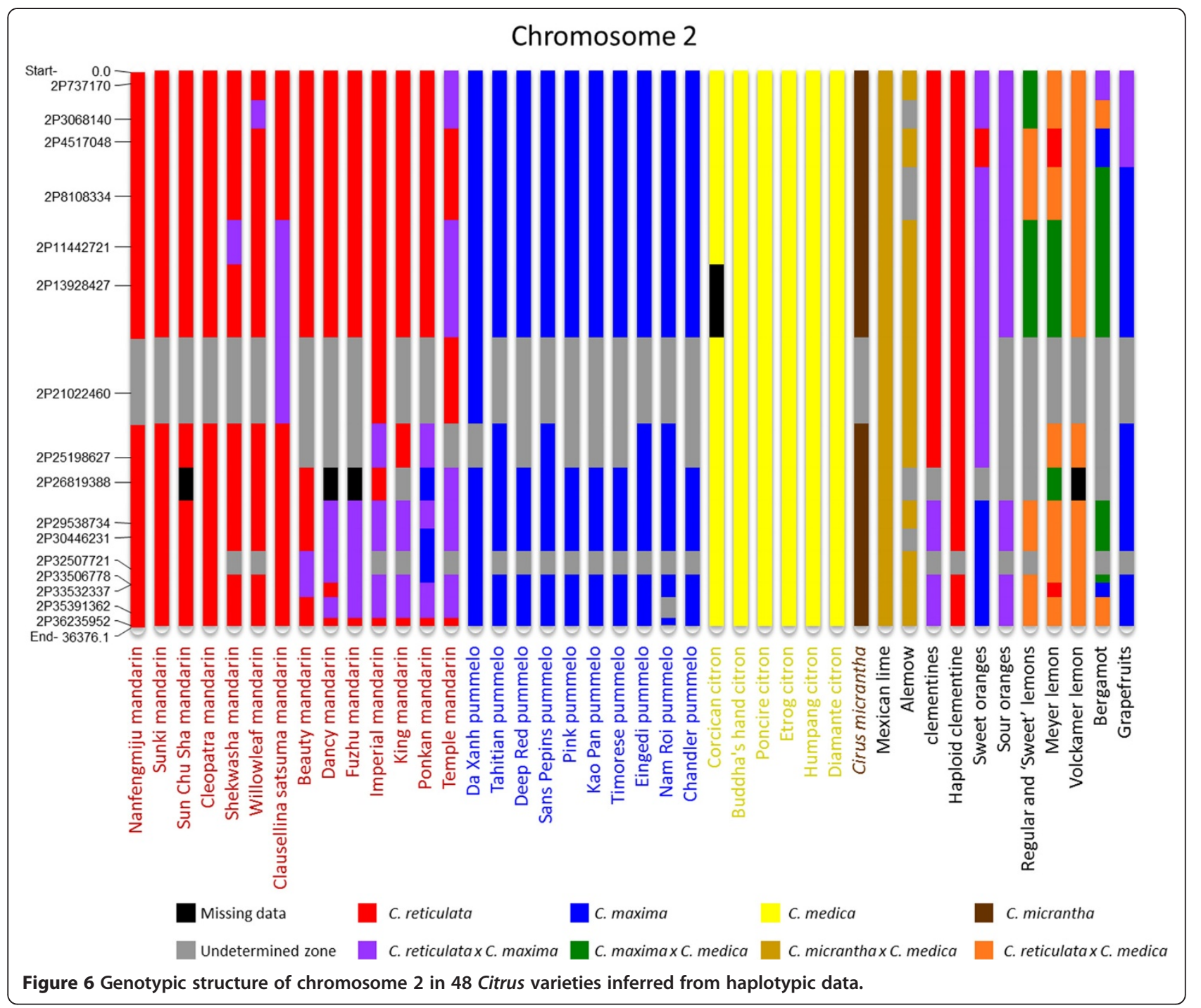

The representative genotypes of the pummelo and citron horticultural groups appeared to be pure $C$. maxima and C. medica, respectively, and no interspecific introgressions were identified. Similarly, no evidence of introgression was found in C. micrantha. Conversely, evidence of introgression by C. maxima was found in 10 of the 14 mandarins studied. This corresponds with recent research [43] in which WGS analysis of 'Willowleaf' and

Table 7 Intra- and interspecies group dissimilarity (average number of SNP/kb between two varieties) after elimination of introgressed haplotypes

\begin{tabular}{llll}
\hline & C. reticulata & C. maxima & C. medica \\
\hline C. reticulata & $2.85^{*}$ & & \\
C. maxima & 11.15 & $1.86^{*}$ & \\
C. medica & 14.80 & 11.21 & $1.24^{*}$ \\
C. micrantha & 13.82 & 10.61 & 12.19 \\
\hline
\end{tabular}

*Average number of SNP/kb at intra-specific level.
'Ponkan' mandarins demonstrated introgression in theses varieties considered to be true mandarins by citrus taxonomists. Three of the four mandarin varieties lacking evidence for introgression ('Cleopatra', 'Sunki', and 'Sun Chu Sha') are used mostly as rootstock and do not share the edible mandarin mitotype revealed by Froelicher et al. [66]. This particular mandarin clade should, therefore, probably not be considered as ancestral to modern cultivated mandarins. The fourth mandarin ('Nanfengmiju') without evidence for introgression shares the cytoplasm of edible mandarins.

The parentage hypothesis of some important commercial species and cultivars suspected to have arisen from reticulate evolution was checked by analyzing the haplotype phylogeny for each gene fragment [Additional file 10].

Citrus sinensis (sweet oranges) and Citrus aurantium (sour oranges): phenotypic data [20] and molecular marker studies $[18,67,68]$ suggested that these two species derived from hybridizations between the C. maxima 
and $C$. reticulata gene pools. Both species have $C$. maxima maternal phylogeny as determined by chloroplast [69] and mitochondrial genome analysis [66]. In the present haplotype analysis within chromosome 2, sour orange displayed $C$. maxima/C. reticulata heterozygosity for each gene fragment. Sweet orange displayed C. reticulata/C. reticulata and C. maxima/C. maxima genome regions in addition to C. maxima/C. reticulata heterozygosity. The presence of a C. maxima/C. maxima region at the end of chromosome 2 disproves the hypothesis of a $(C$. maxima $\times C$. reticulata $) \times C$. reticulata ancestry proposed by Roose et al. [70] from SSR data, and Xu et al. [42] from WGS data. This was also determined by examination of two genes by Garcia-Lor et al. [16] and confirmed by whole genome resequencing data from the ICGC [43]. These results suggest a possible direct F1 interspecific origin for sour orange and a more complex origin for sweet orange that would involve two parents each with $C$. reticulata and C. maxima admixture. These conclusions are in agreement with those proposed by the ICGC [43]. Considering that many mandarin cultivars are introgressed by $C$. maxima, a backcross model of (pummelo $\times$ mandarin) $\times$ mandarin rather than $(C$. maxima $\times C$. reticulata $) \times C$. reticulata would reconcile the $\mathrm{Wu}$ et al. [43] and $\mathrm{Xu}$ et al. [42] hypotheses. For 8 of the 16 gene fragments analyzed in the present study, both sweet orange and sour orange were heterozygous but did not share haplotypes, therefore discarding the hypothesis of a direct relationship between them.

Clementine: It is generally agreed that, a little more than one century ago in Algeria, Father Clement selected clementine as a chance seedling from a 'Mediterranean' mandarin ('Willowleaf'). Previous molecular studies suggested that clementine was a mandarin $\times$ sweet orange hybrid $[13,17,18,71]$, and this was recently confirmed by WGS analysis [43]. From the haplotype data, the larger part of chromosome 2 in clementine appears to be inherited from $C$. reticulata, with $C$. maxima/C. reticulata heterozygosity at the end of the orientated chromosome (phytozome.net [46]) in agreement with WGS data [43]. The haplotype alleles of clementine, sweet orange, and 'Willowleaf' mandarin are in complete agreement with the hypothesis of a 'Willowleaf' $\times$ sweet orange origin.

C. paradisi (grapefruits): The origin of grapefruit is attributed to a natural hybridization between pummelo (C. maxima) and sweet orange (C. sinensis) in the Caribbean after the discovery of the New World by Christopher Columbus [15,18,72,74]. The haplotype analyses agree with this hypothesis, showing coherent haplotypes for most of the gene fragments. In grapefruit, only one fragment (2P32507721) displayed a haplotype observed neither in sweet orange nor in the pummelo accessions (nor in other basic species clusters). However, this gene fragment displayed insufficient differentiation to allow full phylogenetic assignation, and the unassigned grapefruit haplotype may have been inherited from a pummelo not included in our limited samples. Chromosome 2 of grapefruit is mainly inherited from C. maxima and displays a small region of C. maxima/C. reticulata heterozygosity at the start of the scaffold.

Citrus limon (lemons): Based on RFLP, RAPD, and CAPS data, Nicolosi et al. [13] proposed that "regular lemons" arose from hybridization between $C$. aurantium and C. medica. This hypothesis was supported by nuclear SSR [15] and SNP [18] analyses. Moreover, the maternal C. aurantium parentage was confirmed by study of mitochondrial indels [66]. In the present study, 'Eureka,'Lisbon', and 'Sweet' lemon varieties were highly heterozygous and identical. These lemons are very likely somatic mutants of the same hybrid ancestor. The three lemons display successive genome regions with $C$. reticulata/C. medica or $C$. maxima/C. medica heterozygosities. The haplotype allele analysis completely concurs with the sour orange $\times$ citron hypothesis. Indeed, systematic haplotype sharing between lemon and sour orange and the location of the second haplotypes within C. medica clusters were observed. 'Meyer' lemon also appeared to be of tri-specific hybrid origin [15] and displayed C. maxima/C. medica and $C$. reticulata/C. medica heterozygosity, as well as two gene fragments homozygous for a C. reticulata haplotype. Even if the 'Meyer' lemon were found to have a sweet orangelike mitotype [66], as there were only two shared haplotypes between sweet orange and Meyer lemon over the 16 gene fragments, the haplotype analysis disproved the hypothesis that sweet orange was the female parent. 'Volkamer' lemon fragment gene haplotypes suggest that this genotype was a direct hybrid of $C$. reticulata and C. medica.

Citrus aurantifolia ('Mexican' lime,'Alemow', and bergamot): These three citrus types were considered to be distinct species, namely, C. aurantifolia, C. macrophylla, and C. bergamia respectively, by Tanaka [10]. 'Mexican' lime and 'Alemow' displayed interspecific heterozygosity between haplotypes of the $C$. medica and the $C$. micrantha clusters. For 'Mexican' lime, exact haplotype sharing with the analyzed $C$. micrantha sample was found for 15 of the 16 gene fragments. This is in agreement with the hypothesis proposed by Nicolosi et al. [13] that suggests 'Mexican' lime is a C. micrantha $\times C$. medica hybrid. Maternal phylogeny was recently confirmed by mitochondrial marker analysis [66]. Similar results were observed for 'Alemow'. However, exact haplotype correspondence with the analyzed C. micrantha sample was found only for 12 gene fragments. This suggests that the maternal parent of 'Alemow' was closely related to the analyzed C. micrantha, which is in agreement with the Swingle and Reece [9] description of 'Alemow' as a possible hybrid of Citrus celebica Koord (a papeda distinct from 
C. micrantha) or some other species of the subgenus Papeda, with a species of the subgenus Citrus. In 1811, Gallesio [75] proposed that bergamot was a hybrid between lemon and sour orange. However, alternative hypotheses were proposed based on molecular studies. Chen et al. [76] suggested that bergamot could be a hybrid between citron and lime, Herrero et al. [65] and Federici et al. [77] proposed hybridization between sour orange and sweet lime, and hybridization between sour orange and citron was proposed by Nicolosi et al. [13] and Li et al. [78]. The present haplotypic analysis disproved the hypotheses of hybridization between sour orange and citron, and between lemon and 'Mexican' lime, because bergamot displayed haplotypes not found in any of these theoretical parents.

\section{Implications for secondary species breeding}

Some secondary apomictic species such as $C$. aurantium (C. maxima $\times C$. reticulata) and $C$. aurantifolia ( $C$. micrantha $\times$ C. medica), or genotypes such 'Volkamer' lemon $(C$. reticulata $\times C$. medica), displayed interspecific heterozygosity for each gene fragment. They may have resulted directly from reticulation without further sexual recombination. For such secondary species, innovative "like species" cultivars should be searched by direct hybridisation between the ancestral corresponding parental taxa, focusing on germplasm providing the suitable tolerance or resistance traits.

Conversely, other secondary species such as $C$. sinensis and C. limon ("Regular lemon" types) displayed more complex chromosome structures that testified to further interspecific recombination after the first reticulation events. For example, lemons ('Eureka,' 'Lisbon', and 'Sweet' cultivars) systematically had one of their haplotypes within the $C$. medica cluster and the other in either the $C$. maxima or the $C$. reticulata cluster. Under our hypothesis of a sour orange $\times$ citron origin, the changes between C. reticulata/C. medica and C. maxima/C. medica heterozygosities along the chromosome suggest that at least three interspecific crossing over events occurred to produce the sour orange gamete that generated the lemon prototype. Previous studies $[73,78]$ and the present work demonstrated that grapefruit resulted from hybridization between pummelo and sweet orange. For these three important citrus horticultural groups, it will be necessary to have a complete view of the nine chromosome admixture organizations to be able to rebuild similar genomic admixture structures from germplasm. Of these, "regular lemons" should be the simplest to assess despite the three-taxa structure, as it likely resulted from a relatively straightforward sequence of interspecific hybridizations $($ C. maxima $\times$ C. reticulata $) \times$ C. medica). Genomic-assisted selection within progenies resulting from these crossing schemes should allow selection of very close interspecific mosaic structures. Such crossing will, however, be more complex for sweet orange and grapefruit because the two parents of sweet orange were themselves of interspecific origin. However, adequate pre-breeding at the parental level and genomic selection schemes over two or three generations should allow the reconstruction of similar interspecific mosaic genome structures from C. maxima and C. reticulata germplasm alongside desired resistance traits.

\section{Conclusion}

Sixteen gene fragments on chromosome 2 were sequenced in 48 genotypes using 454 amplicon sequencing. The length of the reads and the level of differentiation between the ancestral taxa of modern citrus allowed efficient haplotype phylogenetic assignments for most gene fragments. The analysis of admixture genomic structures of modern species and cultivars revealed C. maxima introgressions in most modern mandarin cultivars. The haplotype results corresponded with previous hypotheses regarding the origin of many secondary citrus species, and provided a novel interpretation for the evolution of chromosome 2. Haplotyping of well-dispersed genome fragments should prove to be widely applicable, particularly for the analysis of evolutionary patterns within gene pools that experienced reticulate evolution. It is clear that this and other NGS methods will dramatically change methods of phylogenetic analysis. Regarding citrus breeding, the interspecific mosaic structure of all nine chromosome should be pursued, as this will provide the opportunity to rebuild the secondary species genomes from ancestral taxa bearing desirable traits.

\section{Additional files}

Additional file 1: Excel table of varieties by common horticultural group and scientific names.

Additional file 2: Excel table presenting information of amplicon location (physical and genetic), annotation of genes, and specific primers for Fluidigm amplification.

Additional file 3: Excel table of multiplex genotype identifiers (MID) and related genotypes.

Additional file 4: Excel table of the distribution of read numbers per gene fragment and varieties for the first Fluidigm run.

Additional file 5: Excel table of the distribution of read numbers per gene fragment and varieties for the two Fluidigm runs, and solutions used for insufficient read number situations.

Additional file 6: Excel table of parameters of SNP genetic diversity for each SNP position.

Additional file 7: Excel table of the Heterozygosity ( $\mathrm{Ho})$ of secondary species.

Additional file 8: Pdf document presenting the analyse of ten Structure software runs at $K=4$. Figure $S 1: 10$ independent Structure software run clusters output permuted and aligned in order to match up as closely as possible. Table S1: Average values of the Ten Structure runs at $\mathrm{K}=4$ for each cluster of each variety (confidence interval estimated with alpha $=0.05)$. 


\section{Additional file 9: Pdf document demonstrating linkage disequilibrium (LD) between SNPs on chromosome 2 .}

Additional file 10: Pdf document demonstrating the maximum likelihood phylogenetic tree of the haplotypic data of the 2P35391362 gene fragment.

Additional file 11: Pdf document demonstrating the observed inherited haplotypic structure of secondary species.

Additional file 12: Excel table of the haplotypic structure of each accession.

Additional file 13: Pdf document demonstrating a 3D distribution of gene sequence SNPs according to their haplotypic $G_{S T}$ value; a: $\mathrm{G}_{\text {sT }}$ value for three horticultural groups (mandarins, pummelos and citrons); $b_{\mathrm{ST}}$ values for three basic taxa after introgression information corrections.

Additional file 14: Excel table of SNP $\mathrm{G}_{\mathrm{ST}}$ values of each taxa before and after introgression information correction.

\section{Competing interests}

The authors declare that they have no competing interests.

\section{Authors' contributions}

FC and GA performed target genomic fragment selection, primer design, amplicon library preparation, genetic analysis of the SNP data, molecular genetic studies, and drafted the manuscript. AGL, FL, XP, and JPJC participated in target genomic fragment selection, and molecular genetic studies. LN participated in the design of the study and its coordination. PO conceived the study, and participated in its design and coordination, and drafted the manuscript. All authors read and approved the final manuscript.

\section{Acknowledgments}

This work was supported by a grant (AGL2011-26490) from the Ministry of 'Economía y Competitividad'- 'Fondo Europeo de Desarrollo Regional' (FEDER) and a grant (Prometeo II/2013/008) from the Generalitat Valenciana, Spain.

We gratefully acknowledge David Karp for his help reviewing the manuscript.

\section{Author details}

'UMR AGAP, Institut National de la Recherche Agronomique (Inra), Centre Inra de Corse, F-20230 San Giuliano, France. ${ }^{2}$ Centro de Protección Vegetal y Biotecnología, Instituto Valenciano de Investigaciones Agrarias (IVIA), 46113 Moncada, Valencia, Spain. ${ }^{3}$ UMR AGAP, Centre de coopération Internationale en Recherche Agronomique pour le Développement (CIRAD), TA A-108/02, 34398 Montpellier, Cedex 5, France.

Received: 21 August 2014 Accepted: 11 December 2014

Published online: 29 December 2014

\section{References}

1. FAO: FAOSTAT http://faostat3.fao.org/home/E. 2014,

2. Ollitrault $P$, Navarro L: Citrus. In Fruit Breeding, Dordrecht Heildlberg edition. Edited by Badenes M, Byrne D. London: Springer New York; 2012:623-662.

3. Wang N, Trivedi P: Citrus Huanglongbing: a newly relevant disease presents unprecedented challenges. Phytopathology 2013, 103(7):652-665.

4. Grosser JW, Dutt M, Omar A, Orbovic V, Barthe G: Progress towards the development of transgenic disease resistance in citrus. Acta Hort (ISHS) 2011, 892(101):107.

5. Texeira DC, Ayres J, Kitajima EW, Danet L, Jagoueix-Eveillard S, Saillard C, Bové JM: First Report of a Huanglongbing-Like Disease of Citrus in Sao Paulo State, Brazil and Association of a New Liberibacter Species, "Candidatus Liberibacter americanus", with the Disease. Plant Dis 2005, 89(1):107

6. Grosser JW, Deng XX, Goodrich RM: Somaclonal variation in sweet orange: practical applications for variety improvement and possible causes. In Citrus genetics, breeding and biotechnology. Edited by Kham IA. Wallingford: CAB International; 2007:219-233.

7. Krueger RR, Navarro L: Citrus germplasm resources. In Citrus Genetics, Breeding and Biotechnology. Anonymous Wallingford, UK: CAB International; 2007:45-140.
8. Scora RW: On the history and origin of Citrus. Bull Torrey Bot Club 1975, 102:369-375

9. Swingle WT, Reece PC: The botany of Citrus and its wild relatives. In The citrus industry. Volume 1. 2nd edition. Edited by Reuther W, Webber HJ, Batchelor LD. Berkeley, California, USA: University of California Press; 1967:190-430

10. Tanaka T: Citologia: Semi-centennial Commemoration Papers on Citrus Studies. Osaka: Citologia Supporting Foundation; 1961.

11. Mabberley DJ: A classification for edible Citrus (Rutaceae). Telopea 1997, 7(2):167-172.

12. Federici $C T$, Fang DQ, Scora RW, Roose ML: Phylogenetic relationships within the genus Citrus (Rutaceae) and related genera as revealed by RFLP and RAPD analysis. Theor App/ Genet 1998, 96(6/7):812-822.

13. Nicolosi E, Deng ZN, Gentile A, La Malfa S, Continella G, Tribulato E: Citrus phylogeny and genetic origin of important species as investigated by molecular markers. Theor Appl Genet 2000, 100:1155-1166.

14. Barkley NA, Roose ML, Krueger RR, Federici CT: Assessing genetic diversity and population structure in a citrus germplasm collection utilizing simple sequence repeat markers (SSRs). Theor Appl Genet 2006, 112(8):1519-1531.

15. Garcia-Lor A, Luro F, Navarro L, Ollitrault P: Comparative use of InDel and SSR markers in deciphering the interspecific structure of cultivated citrus genetic diversity: a perspective for genetic association studies. Mol Genet Genomics 2012, 287(1):77-94.

16. Garcia-Lor A, Curk F, Snoussi-Trifa H, Morillon R, Ancillo G, Luro F, Navarro L, Ollitrault P: A nuclear phylogenetic analysis: SNPs, indels and SSRs deliver new insights into the relationships in the 'true citrus fruit trees' group (Citrinae, Rutaceae) and the origin of cultivated species. Ann Bot 2013, 111(1):1-19.

17. Ollitrault $P$, Terol J, Chen C, Federici CT, Lotfy S, Hippolyte I, Ollitrault F, Berard A, Chauveau A, Cuenca J, Costantino G, Kacar Y, Mu L, Garcia-Lor A, Froelicher Y, Aleza P, Boland A, Billot C, Navarro L, Luro F, Roose ML, Gmitter $F G$, Talon $M$, Brunel D: A reference genetic map of $C$. clementina hort. ex Tan.; citrus evolution inferences from comparative mapping. BMC Genomics 2012, 13:593-2164-13-593.

18. Ollitrault P, Terol J, Garcia-Lor A, Berard A, Chauveau A, Froelicher Y, Belzile C, Morillon R, Navarro L, Brunel D, Talon M: SNP mining in C. clementina $\mathrm{BAC}$ end sequences; transferability in the Citrus genus (Rutaceae), phylogenetic inferences and perspectives for genetic mapping. BMC Genomics 2012, 13:13-2164-13-13.

19. Webber HJ, Reuther W, Lawton HW: istory and development of the Citrus industry. In The Citrus Industry, Volume Volume 1. Edited by Reuther W, et al. Berkeley: University of California, Division of Agricultural Sciences; 1967:1-39.

20. Barrett HC, Rhodes AM: A numerical taxonomic study ofaffinity relationships in cultivated Citrus and its close relatives. Syst Bot 1976, 1:105-136.

21. Ollitrault $P$, Jacquemond C, Dubois C, Luro F: Citrus. In Genetic diversity of cultivated tropical plants. Edited by Hamon P, Seguin M, Perrier X, Glaszmann J-C. Montpellier: Cirad; 2003:193-217.

22. Luro F, Gatto J, Costantino G, Pailly O: Analysis of genetic diversity in Citrus. Plant Genetic Resources 2011, 9:218-221.

23. Fanciullino AL, Dhuique-Mayer C, Luro F, Casanova J, Morillon R, Ollitrault P: Carotenoid diversity in cultivated citrus is highly influenced by genetic factors. J Agric Food Chem 2006, 54(12):4397-4406.

24. Stebbins G: Variation and evolution in plants: Columbia University Press ed. New York: Columbia University Press; 1950.

25. Grant V: Plant Speciation: 2nd Edit. ed. New York: Colombia University Press; 1981.

26. Arnold ML: Natural hybridization and evolution. New York: Oxford University Press; 1997.

27. Doolittle WF: Phylogenetic Classification and the Universal Tree. Science 1999, 284(5423):2124-2129.

28. Otto SP, Whitton J: Polyploid incidence and evolution. Annu Rev Genet 2000, 34:401-437.

29. Linder $\mathrm{CR}$, Rieseberg $\mathrm{LH}$ : Reconstructing patterns of reticulate evolution in plants. Am J Bot 2004, 91:1700-1708.

30. Pamilo $\mathrm{P}$, Nei M: Relationships between gene trees and species trees. Mol Biol Evol 1988, 5:568-583.

31. Rieseberg LH, Soltis DE: Phylogenetic consequences of cytoplasmic gene flow in plants. Trends in Plants 1991, 5:65-84. 
32. Beiko RG, Hamilton N: Phylogenetic identification of lateral genetic transfer events. BMC Evol Biol 2006, 6:15.

33. Rieseberg LH, Sinervo B, Linder CR, Ungerer MC, Arias DM: Role of gene interactions in hybrid speciation: evidence from ancient and experimental hybrids. SCIENCE-NEW YORK THEN WASHINGTON- 1996, 272:741-744.

34. Rieseberg LH, Raymond O, Rosenthal DM, Lai Z, Livingstone K, Nakazato T, Durphy JL, Schwarzbach AE, Donovan LA, Lexer C: Major ecological transitions in annual sunflowers facilitated by hybridization. Science 2003, 301:1211-1216.

35. Rousseau-Gueutin M, Gaston A, Aïnouche A, Aïnouche ML, Olbricht K, Staudt G, Richard L, Denoyes-Rothan B: Tracking the evolutionary history of polyploidy in Fragaria L. (strawberry): new insights from phylogenetic analyses of low-copy nuclear genes. Mol Phylogenet Evol 2009, 51(3):515-530

36. Fortune P, Pourtau N, Viron N, Ainouche M: Molecular phylogeny and reticulate origins of the polyploid Bromus species from section Genea (Poaceae). Am J Bot 2008, 95(4):454-464.

37. Ramadugu C, Pfeil BE, Keremane ML, Lee RF, Maureira-Butler IJ, Roose ML: A six nuclear gene phylogeny of Citrus (Rutaceae) taking into account hybridization and lineage sorting. PLoS One 2013, 8(7):e68410.

38. Maddison WP, Knowles LL: Inferring phylogeny despite incomplete lineage sorting. Syst Biol 2006, 55(1):21-30.

39. Jumpponen A, Jones KL: Massively parallel 454-sequencing of Quercus macrocarpa phyllosphere fungal communities indicates reduced richness and diversity in urban environments. New Phytol 2009, 184:438-448.

40. Sønstebø JH, Gielly L, Brysting AK, Elven R, Edwards M, Haile J, Willersleve E, Coissac E, Rioux D, Sannier J, Taberlet P, Brochmann C: Using nextgeneration sequencing for molecular reconstruction of past Arctic vegetation and climate. Mol Ecol Resour 2010, 10(6):1009-1018.

41. Taudien S, Groth M, Huse K, Petzold A, Szafranski K, Hampe J, Rosenstiel P, Schreiber S, Platzer M: Haplotyping and copy number estimation of the highly polymorphic human beta-defensin locus on 8 p23 by 454 amplicon sequencing. BMC Genomics 2010, 11(1):252.

42. Xu Q, Chen L, Ruan X, Chen D, Zhu A, Chen C, Bertrand D, Jiao W, Hao B, Lyon PM, Chen J, Gao S, Xing F, Lan H, Chang J, Ge X, Lei Y, Hu Q, Miao Y, Wang L, Xiao S, Biswas KM, Zeng W, Guo F, Cao H, Yang X, Xu X, Cheng Y, $X u$ J, Liu J, et al: The draft genome of sweet orange (Citrus sinensis). Nat Genet 2013, 45:59-66.

43. Wu GA, Prochnik S, Jenkins J, Salse J, Hellsten U, Murat F, Perrier X, Ruiz M, Scalabrin S, Terol J, Takita MA, Labadie K, Poulain J, Couloux A, Jabbari K, Cattonaro F, Del Fabbro C, Pinosio S, Zuccolo A, Chapman J, Grimwood J, Tadeo FR, Estornell LH, Munoz-Sanz JV, Ibanez V, Herrero-Ortega A, Aleza P, Perez-Perez J, Ramon D, Brunel D, et al: Sequencing of diverse mandarin, pummelo and orange genomes reveals complex history of admixture during citrus domestication. Nat Biotechnol 2014, 32(7):656-662.

44. Okou DT, Steinberg KM, Middle C, Cutler DJ, Albert TJ, Zwick ME: Microarray-based genomic selection for high-throughput resequencing Nat Methods 2007, 4(11):907-909.

45. Bybee SM, Bracken-Grissom H, Haynes BD, Hermansen RA, Byers RL, Clement MJ, Udall JA, Wilcox ER, Crandall KA: Targeted amplicon sequencing (TAS): a scalable next-gen approach to multilocus, multitaxa phylogenetics. Genome Biol Evol 2011, 3:1312-1323.

46. Department of Energy's Joint Genome Institute, Center for Integrative Genomics: Phytozome; http://www.phytozome.net/. 2014.

47. Fluidigm Corp.: Access Array TM System; http://www.fluidigm.com/accessarray-system.html; 2014.

48. Curk, F.;Ancillo, G.;Garcia-Lor, A.;Luro, F.;Navarro, L.;Ollitrault, P.; Multilocus SNPs analysis allows phylogenetic assignation of DNA fragments to decipher the interspecific mosaic genome structure of cultivated citrus; Plant Genome Evolution 2011 , 4-6 Sep 2011, P2.1572 [http://f1000.com/posters/browse/summary/1089299]

49. Life Sciences Corp.: Using Multiplex Identifier (MID) Adaptors for the GS FLX Titanium Chemistry - Extended Mid Set. Technical Bulletin Genome Sequencer FLX System 2009, 005:1-7.

50. Life Sciences Corp.: Amplicon Fusion Primer Design Guidelines for GS FLX Titanium Series Lib-A Chemistry. Technical Bulletin Genome Sequencer FLX System 2009, 013:1-3.

51. PRINSEQ: PReprocessing and INformation of SEQuence data: Easy and rapid quality control and data preprocessing; http://prinseq.sourceforge. net/index.html. 2012
52. Life Sciences Corp.: 454 Sequencing System Software Manual, v 2.5.3; Part C - GS De Novo Assembler, GS Reference Mapper, SFF Tools. 454 Sequencing System Software Manual 2010:2-213.

53. DNASTAR Inc.: DNASTAR Sofware for life Scientists; http://www.dnastar com/t-nextgen-seqman-ngen.aspx. 2014.

54. Wright S: Variability Within and Among Natural Populations. In Evolution and the Genetics of Populations. Volume 4. Anonymous Chicago, IL: The University of Chicago Press; 1978.

55. Belkhir K, Borsa P, Chikhi L, Raufaste N, Bonhomme F: GENETIX 4.05, logiciel sous Windows TM pour la génétique des populations. Laboratoire Génome, Populations, Interactions, CNRS UMR 5000 1996-2004, (Université de Montpellier II, Montpellier (France).).

56. Dereeper A, Nicolas S, Lecunff L, Bacilieri R, Doligez A, Peros JP, Ruiz M This P: SNiPlay: a web-based tool for detection, management and analysis of SNPs. Application to grapevine diversity projects. BMC Bioinformatics 2011, 12(1):134

57. Dereeper A, Nicolas S, Lecunff L, Bacilieri R, Doligez A, Peros JP, Ruiz M, This P: http://sniplay.cirad.fr/cgi-bin/home.cgi. 2013, 2014

58. Perrier $X$, Jacquemoud-Collet JP: DARwin software. 2006, (http://darwin cirad.fr/).

59. Tamura K, Stecher G, Peterson D, Filipski A, Kumar S: MEGA6: Molecular Evolutionary Genetics Analysis version 6.0. Mol Biol Evol 2013, 30(12):2725-2729.

60. Van Berloo R: GGT 2.0: versatile software for visualization and analysis of genetic data. J Hered 2008, 99(2):232-236.

61. Pritchard Lab SU: Structure Software; http://pritchardlab.stanford.edu/ structure.html 2014

62. Pritchard JK, Stephens M, Donnelly P: Inference of population structure using multilocus genotype data. Genetics 2000, 155(2):945-959.

63. Falush D, Stephens M, Pritchard JK: Inference of population structure using multilocus genotype data: linked loci and correlated allele frequencies. Genetics 2003, 164(4):1567-1587.

64. Evanno G, Regnaut S, Goudet J: Detecting the number of clusters of individuals using the software STRUCTURE: a simulation study. Mol Ecol 2005, 14(8):2611-2620.

65. Herrero R, Asins MJ, Pina JA, Carbonell EA, Navarro L: Genetic diversity in the orange subfamily Aurantioideae. II. Genetic relationships among genera and species. Theor App/ Genet 1996, 93(8):1327-1334.

66. Froelicher Y, Mouhaya W, Bassene JB, Costantino G, Kamiri M, Luro F, Morillon R, Ollitrault P: New universal mitochondrial PCR markers reveal new information on maternal citrus phylogeny. Tree Genetics and Genomes 2011, 7(1):49-61.

67. Uzun A, Yesiloglu T, Polat I, Aka-Kacar Y, Gulsen O, Yildirim B, Tuzcu O, Tepe S, Canan I, Anil S: Evaluation of Genetic Diversity in Lemons and Some of Their Relatives Based on SRAP and SSR Markers. Plant Mol Biol Report 2011, 29(3):693-701.

68. Uzun A, Yesiloglu T, Aka-Kacar Y, Tuzcu O, Gulsen O: Genetic diversity and relationships within Citrus and related genera based on sequence related amplified polymorphism markers (SRAPs). Sci Hortic 2009, 121(3):306-312.

69. Bayer RJ, Mabberley DJ, Morton C, Miller CH, Sharma IK, Pfeil BE, Rich S, Hitchcock R, Sykes S: A molecular phylogeny of the orange subfamily (Rutaceae: Aurantioideae) using nine cpDNA sequences. Am J Bot 2009, 96(3):668-685.

70. Roose ML, Federici CT, Mu L, Kwok K, Vu C: Map-based ancestry of sweet orange and other citrus variety groups. Gentile A, Tribulato E eds Second International Citrus Biotechnology Symposium, 28 Tremestieri Etneo. Italy: Emme Erre Grafica; 2009.

71. Ollitrault F, Terol J, Pina JA, Navarro L, Talon M, Ollitrault P: Development of SSR markers from Citrus clementina (Rutaceae) BAC end sequences and interspecific transferability in Citrus. Am J Bot 2010, 97(11):e124-e129.

72. Webber HJ: Cultivated varieties of citrus. In The Citrus Industry. History World Distribution, Botany andVarieties, Volume Volume 1. University of California Press edition. Edited by Anonymous California. ; 1943:475-668.

73. de Moraes A, dos Santos Soares Filho W, Guerra M: Karyotype diversity and the origin of grapefruit. Chromosome Research 2007, 15(1):115-121.

74. Scora RW, Kumamoto J, Soost RK, Nauer EM: Contribution to the origin of the grapefruit Citrus paradisi (Rutaceae). Syst Bot 1982, 7:170-177.

75. Gallesio G: Traité du citrus: Louis Fantin ed. Paris: Chez Louis Fantin Libraire; 1811

76. Chen LG, Omura M, Hidaka T: A study on the taxonomy of citrus with GOT isozymes. Acta Horticulturae Sinica 1991, 18(1):27-32. 
77. Federici $C$, Roose ML, Scora RW: RFLP analysis of the origin of Citrus bergamia, Citrus jambhiri, and Citrus limonia. Acta Horticult 2000, 535:55-62.

78. Li X, Xie R, Lu Z, Zhou Z: The origin of cultivated citrus as inferred from internal transcribed spacer and chloroplast DNA sequence and amplified fragment length polymorphism fingerprints. J Am Soc Hortic Sci 2010, 135(4):341-350.

Submit your next manuscript to BioMed Central and take full advantage of:

- Convenient online submission

- Thorough peer review

- No space constraints or color figure charges

- Immediate publication on acceptance

- Inclusion in PubMed, CAS, Scopus and Google Scholar

- Research which is freely available for redistribution 